\title{
Discovery of late-time X-ray flare and anomalous emission line enhancement after the nuclear optical outburst in a narrow-line Seyfert 1 Galaxy
}

\author{
W. J. Zhang ${ }^{1}$, X. W. Shu ${ }^{1}$, Z. F. Sheng ${ }^{2}$, L. M. Sun ${ }^{1}$, L. M. Dou ${ }^{3}$, N. Jiang ${ }^{2}$, J. G. Wang ${ }^{4}$, X. Y. Hu ${ }^{2}$, Y. B. Wang ${ }^{2}$, and \\ T. G. Wang ${ }^{2}$
}

${ }^{1}$ Department of Physics, Anhui Normal University, Wuhu, Anhui, 241002, China e-mail: xwshu@mail . ahnu.edu.cn

2 CAS Key Laboratory for Researches in Galaxies and Cosmology, Department of Astronomy, University of Science and Technology of China, Hefei, Anhui 230026, China e-mail: shengzf@ustc.edu.cn; jnac@ustc.edu.cn

3 Department of Astronomy, Guangzhou University, Guangzhou 510006, China

4 Yunnan Observatories, Chinese Academy of Sciences, Kunming 650011, China

January 27, 2022

\begin{abstract}
CSS J102913+404220 is an atypical narrow line Seyfert 1 galaxy with an energetic optical outburst occurred in coincidence with its nucleus. We present a detailed analysis of its multi-wavelength photometric and spectroscopic observations covering a period of decade since outburst. We detect mid-infrared (MIR) flares delayed by about two months relative to the optical outburst, with an extremely high peak luminosity of $L_{4.6 u m}>10^{44} \mathrm{erg} \mathrm{s}^{-1}$. The MIR peak luminosity is at least an order of magnitude higher than any known supernovae explosions, suggesting the optical outburst might be due to a stellar tidal disruption event (TDE). We find late-time $\mathrm{X}$-ray brightening by a factor of $\gtrsim 30$ with respect to what is observed about 100 days after the optical outburst peak, followed by a flux fading by a factor of $\sim 4$ within two weeks, making it one of Active Galactic Nuclei (AGNs) with extreme variability. Despite the dramatic X-ray variability, there are no coincident strong flux variations in optical, UV and MIR bands. This unusual variability behavior has been seen in other highly accreting AGNs and could be attributed to absorption variability. In this scenario, the decrease in the covering factor of absorber with accretion rate could cause the X-ray brightening, possibly induced by the TDE. Most strikingly, while the UV/optical continuum remains little changes with time, an evident enhancement in the flux of $\mathrm{H} \alpha$ broad emission line is observed, about a decade after the nuclear optical outburst, which is an anomalous behavior never seen in any other AGNs. Such an $\mathrm{H} \alpha$ anomaly could be explained by the replenishment of gas clouds and excitation within Broad Line Region (BLR) that originates, perhaps from the interaction of outflowing stellar debris with BLR. The results highlight the importance of late-time evolution of TDE that could affect the accreting properties of AGN, as suggested by recent simulations.
\end{abstract}

Key words. galaxies: active—accretion, accretion disks—X-rays: individual (CSS J102913+404220)

\section{Introduction}

It is believed that active galactic nuclei (AGNs) are powered by supermassive black holes (SMBHs, $M_{\mathrm{BH}} \sim 10^{6}-10^{9} M_{\odot}$ ) accreting surrounding material. The optical and UV photons are thought to originate from the accretion disk, and a faction of disk emission transported into the hot corona, presumably in the immediate vicinity of the SMBH, generating powerful X-rays at higher energies. While rapid X-ray variability over a timescale from minutes to hours is ubiquitous in AGNs (Ulrich et al. 1997; González-Martín \& Vaughan 2012), large amplitude variations on longer timescales of years are not common, typically in the range $\sim 50-200 \%$ (Saxton et al. 2011, Yang et al. 2016, Middei et al. 2017, Boller et al. 2021). In addition, exotic AGN X-ray variability characterized by quasi-periodic modulations (Gierliński et al. 2008, Song et al. 2020) or even repeating short-lived X-ray eruptions (Sun et al. 2013; Miniutti et al. 2019) are also found, providing a unique probe to the accretion physics and $\mathrm{X}$ ray radiation mechanisms.

Extreme X-ray variability by orders of magnitude on time scale of years has been found in several types of AGNs (e.g.,
Grupe et al.2012a), some of which are also accompanied by the $\mathrm{X}$-ray spectral changes, i.e., from Compton-thin to Comptonthick or vice versa. This latter case can be naturally explained in terms of variations of absorption column density along the line of sight (e.g., Risaliti et al. 2005, Miniutti et al. 2014). Alternatively the dramatic X-ray flux and spectral variations can be ascribed to the "switch off and on" of the gas accretion onto SMBHs (Guainazzi et al.1998, Gilli et al. 2000). However, such a scenario is difficult to reconcile with the observations of $\mathrm{X}$ ray weak state in several highly accreting narrow line Seyfert 1s (NSy1s) and quasars, where the UV flux remains almost constant despite the huge X-ray drop (Gallo et al. 2011; Miniutti et al. 2012, Grupe et al. 2019). Since sources with high Eddington ratios such as NSy1s are expected to have a geometrically thick accretion disk in innermost region, the disk self-shielding can explain the extreme X-ray variability while UV/optical continuum at larger radii is not affected, similar to the explanation proposed for the X-ray behavior of weak-line quasars (Luo et al. 2015, Ni et al. 2018). Another scenario that has been invoked to account for the X-ray weakness is that the direct nuclear emis- 
sion is suppressed by the light-bending effect near the SMBH (Miniutti et al. 2003, Miniutti \& Fabian 2004).

While extreme X-ray variations appear to be rare among standard AGNs, they are more commonly seen in stellar tidal disruption events (TDE; see Komossa 2015 for a review). Such an event occurs when a star passes sufficiently close to a SMBH and is torn apart when the tidal force of the SMBH exceeds its self-gravity. While roughly half of the stellar material will be ejected, the other half will remain bound and eventually be accreted, producing a luminous flare of electromagnetic radiation (Rees 1988). For a BH mass with $M_{\mathrm{BH}} \sim 10^{6} M_{\odot}$, the peaking luminosity can reach $\sim 10^{44} \mathrm{erg} \mathrm{s}^{-1}$ and then declines by up to factors of $>100$ to the quiescent flux level of the host galaxy. X-ray observations have revealed a population of TDE candidates with such large-amplitude X-ray variability (e.g., Komossa 2015; Lin et al. 2017; Saxton et al. 2019). Note that similar extreme optical outbursts in the centers of galaxies have been detected recently, but very few of them are associated with strong X-ray emission, the nature of which is still under debate (Kankare et al.|2017). In addition, theoretical works have suggested that TDEs can occur in AGNs and the event rate may be even higher than in inactive galaxies (Karas \& Šubr 2007). Indeed, an increasing number of TDE candidates have been found in galaxies with preexisting AGN activity (e.g., van Velzen et al. 2016; Blanchard et al. 2017; Shu et al. 2018, Liu et al. 2020). In this case, however, identifying whether nuclear outburst is due to a TDE or a particular episode of AGN variability is not trivial (Saxton et al. 2015: Auchettl et al. 2018; Trakhtenbrot et al. 2019, Hinkle et al. 2021). On the other hand, in the presence of TDE, the innermost regions of the accretion flow can be affected by the evolution of stellar debris, leading to unique and remarkable AGN X-ray variabilities (Blanchard et al. 2017, Chan et al. 2019, Ricci et al. 2020, 2021).

CSSJ102912.58+404219.7 (CSS1029 hereafter) is a NSy1like galaxy at $z=0.147$ that was discovered by the Catalina Real-time Transient Survey (CRTS; Drake et al. 2009) as an extraordinary optical transient in the galaxy nucleus (Drake et al. 2011; D11 hereafter). Its extremely high peak luminosity of $M_{\mathrm{V}}=-22.7$, distinctive features of hydrogen Balmer emission lines, and slow rise and fall of light curve over a period of months led D11 to classify the transient as a ultra-luminous Type IIn supernovae. However, based on the similar spectral properties to PS16dtm, another luminous transient occurred at the nucleus of a NSy1, Blanchard et al. (2017) suggested that CSS1029 is more like a non-standard TDE (see also Kankare et al. 2017). The recent argument that the super luminous supernova (SLSN) ASASSN-15h is more consistent with a TDE (Leloudas et al. 2016), suggests that the bright optical outburst in CSS1029 may not necessarily be due to SLSN. Here we present new optical spectroscopy as well as archival multi-wavelength observations of CSS1029, and confirm the source as one of most luminous nuclear transients in terms of mid-infrared (MIR) luminosity, making its association with Type IIn SLSN impossible. In particular, we find a bright X-ray flare about five years since optical outburst, while no obvious coordinated photometric variability at other bands is found. Most strikingly, our spectroscopy follow-up observations about decade later reveal a significant flux enhancement in the $\mathrm{H} \alpha$ emission line, while the continuum emission remains almost constant. This kind of $\mathrm{H} \alpha$ variability is anomalous and rare among either TDEs or NSy1s. In Section 2, we describe the observations and data reductions. In Section 3, we present detailed analysis and results. Possible explanations for the late time X-ray brightening and $\mathrm{H} \alpha$ anomaly are given in Section 4.

\section{Observations and data}

CSS1029 was first discovered as a bright optical transient on Feb 172010 (D11). Prompt Swift observations of the source were performed four times in 2010. There are also seven follow-up Swift observations performed on 2015, and two observations on 2020. Details of the observation information were listed in Table 1. Some results from the Swift 2010 observations have been presented in D11, while the data after 2015 have not yet been fully reported. For the Swift /XRT (X-ray Telescope) data, all of them were operated in Photon Counting (PC) mode. After reducing the data following standard procedures by xrtpipline, we used Heasoft (v6.19) to extract spectrum with the task xselect. The source spectrum was extracted in a circular region with a $40^{\prime \prime}$ radius, and we used another source-free circular region with a radius of $100^{\prime \prime}$ for background. We generated ancillary response function files (ARFs) by using xrtmkarf, and the response matrix files (RMFs) were downloaded with the latest calibration files.

For Swift UVOT (the Ultra-violet Optical Telescope) data, all four observations in 2010 have data in six filters (UVW2, UVM2, UVW1, U, B, V). By giving the source coordinate from optical position, we obtained magnitudes in the six bands for each observation by using the task uvotsource. We performed flux extraction for source and background by using a circular region with $5^{\prime \prime}$ radius and a source-free region of circle with $50^{\prime \prime}$ radius, respectively. In observations performed in 2015, only three UV band filters have been used, and the source and background flux were extracted using the same manner as for the 2010 data. All the UVOT magnitudes are referred to the AB magnitude system, and listed in Table 1 . Note that the UVOT measurements are consistent with the results of D11, where only the data taken on 2010 are presented.

We performed follow-up long-slit optical spectroscopy observations of CSS1029, aiming to examine any spectral evolution at later times. The observations were taken twice with the Double Beam Spectrograph (DBSP) on the 200-in Hale telescope at Palomar Observatory (P200) on Feb 2018 and Feb 2020. The latter one is quasi-simultaneously with the recent X-ray observations. We used the D55 dichroic which splits the incoming photons into the $600 / 4000$ (lines/mm) grating for the blue side, and 316/7500 grating for the red side. The grating angles were adjusted so that a nearly continuous wavelength coverage from 3300 to $10000 \AA$ is obtained, with a spectral resolution of $R=\lambda / \Delta \lambda \sim 1080$ at $\lambda \sim 6600 \AA$. The data were reduced following the standard procedures. The default slit width for the DBSP observations is $1.5^{\prime \prime}$. Although the observations have different seeing conditions, i.e., 3.3" for Feb 2018 and 1.5" for Feb 2020, we used the same aperture size of $\sim 1.5^{\prime \prime}$ to extract spectrum, with the aim to mitigate potential effect on flux measurements of continuum and emission lines with different apertures. The spectrum was then flux calibrated using standard star observations from the same night. In addition, we also observed the source with the YFOSC (Yunnan Faint Object Spectrograph and Camera) mounted on the Lijiang $2.4 \mathrm{~m}$ telescope at the Yunnan Observatory on April 2020, Jan 2021 and May 2021, respectively. For the former two YFOSC observations, we used the spectral settings of G8 grating plus a slit width of $2.5^{\prime \prime}$, while for latter a slit width of $1.8^{\prime \prime}$ was used. The observations have been performed under a typical seeing of $\sim 2^{\prime \prime}$. The G8 grating covers a wavelength range from 5100 to $9600 \AA$, with a spectral resolution of $R \sim 380$ at $\lambda \sim 6600 \AA$ for the $2.5^{\prime \prime}$ slit, and $R \sim 810$ for the $1.8^{\prime \prime}$ slit, respectively. The YFOSC data were reduced using standard techniques in IRAF and the same aperture of $1.5^{\prime \prime}$ was used to extract spectrum. Details on the spectroscopic observa- 
Table 1. X-ray count rates, fluxes and optical magnitudes for CSS1029 observed by Swift.

\begin{tabular}{cccccccccc}
\hline \hline $\begin{array}{c}\text { Obs. date } \\
(1)\end{array}$ & $\begin{array}{c}\text { Exp. time } \\
(2)\end{array}$ & $\begin{array}{c}\text { Counts rate } \\
(3)\end{array}$ & $\begin{array}{c}\text { X-ray flux } \\
(4)\end{array}$ & $\begin{array}{c}M_{\text {UVW2 }} \\
(5)\end{array}$ & $\begin{array}{c}M_{\text {UVM2 }} \\
(6)\end{array}$ & $\begin{array}{c}M_{\text {UVW1 }} \\
(7)\end{array}$ & $\begin{array}{c}M_{\mathrm{U}} \\
(8)\end{array}$ & $\begin{array}{c}M_{\mathrm{B}} \\
(9)\end{array}$ & $\begin{array}{c}M_{\mathrm{V}} \\
(10)\end{array}$ \\
\hline $2010-04-06$ & 3490 & $0.31 \pm 0.10$ & $0.97 \pm 0.31$ & $18.06 \pm 0.04$ & $17.87 \pm 0.04$ & $17.56 \pm 0.04$ & $16.78 \pm 0.04$ & $16.35 \pm 0.04$ & $16.12 \pm 0.06$ \\
$2010-04-25$ & 3010 & $0.12 \pm 0.07$ & $0.38 \pm 0.22$ & $18.38 \pm 0.05$ & $18.12 \pm 0.05$ & $17.71 \pm 0.05$ & $17.08 \pm 0.06$ & $16.61 \pm 0.06$ & $16.24 \pm 0.08$ \\
$2010-05-09$ & 3285 & $0.11 \pm 0.07$ & $0.36 \pm 0.22$ & $18.50 \pm 0.06$ & $18.13 \pm 0.05$ & $17.92 \pm 0.06$ & $17.10 \pm 0.07$ & $16.75 \pm 0.07$ & $16.31 \pm 0.09$ \\
$2010-05-23$ & 3985 & $<0.08^{\mathrm{a}}$ & $<0.24^{\mathrm{a}}$ & $18.50 \pm 0.05$ & $18.35 \pm 0.06$ & $17.90 \pm 0.05$ & $17.10 \pm 0.06$ & $16.83 \pm 0.06$ & $16.59 \pm 0.09$ \\
$2015-03-26$ & 175 & $<1.70$ & $<5.30$ & $19.44 \pm 0.20$ & $19.81 \pm 0.31$ & $18.96 \pm 0.21$ & - & - & - \\
$2015-03-28$ & 595 & $3.15 \pm 0.74$ & $9.52 \pm 2.24$ & $19.22 \pm 0.10$ & $19.41 \pm 0.14$ & $19.21 \pm 0.11$ & - & - & - \\
$2015-03-29$ & 1451 & $2.86 \pm 0.45$ & $8.65 \pm 1.36$ & $19.39 \pm 0.18$ & $19.29 \pm 0.22$ & $19.34 \pm 0.19$ & - & - & - \\
$2015-04-02$ & 284 & $<1.05$ & $<3.27$ & $19.43 \pm 0.16$ & $19.47 \pm 0.20$ & $19.18 \pm 0.17$ & - & - & - \\
$2015-04-04$ & 701 & $1.24 \pm 0.43$ & $3.75 \pm 1.30$ & $19.53 \pm 0.20$ & $19.14 \pm 0.20$ & $19.31 \pm 0.19$ & - & - & - \\
$2015-04-05$ & 867 & $0.33 \pm 0.19$ & $0.98 \pm 0.60$ & $19.49 \pm 0.18$ & $19.64 \pm 0.25$ & $18.93 \pm 0.17$ & - & - & - \\
$2015-04-11$ & 571 & $0.87 \pm 0.40$ & $2.63 \pm 1.22$ & $19.60 \pm 0.19$ & $19.91 \pm 0.29$ & $19.14 \pm 0.16$ & - & - & - \\
$2020-01-12$ & 759 & $0.77 \pm 0.32$ & $2.25 \pm 0.91$ & $19.37 \pm 0.13$ & $19.22 \pm 0.11$ & $18.99 \pm 0.15$ & $18.72 \pm 0.17$ & $18.37 \pm 0.20$ & $>18.15$ \\
$2020-02-28$ & 1650 & $<0.27$ & $<0.78$ & $19.17 \pm 0.08$ & $19.27 \pm 0.08$ & $19.03 \pm 0.10$ & $18.50 \pm 0.11$ & $18.73 \pm 0.19$ & $17.79 \pm 0.19$ \\
\hline \hline
\end{tabular}

Notes. Column (1): Swift XRT observing date; Column (2): XRT exposure time in unit of second; Column (3): Count rates in the 0.3-2 keV, in units of $10^{-2} \mathrm{cts} \mathrm{s}^{-1}$; Column (4): X-ray flux in the $0.3-2 \mathrm{keV}$, in units of $10^{-13} \mathrm{erg} \mathrm{cm}^{-2} \mathrm{~s}^{-1}$; Column (5): UVW2 $\left(\lambda_{c}=1928 \AA\right.$ ) magnitude in the AB system; Column (6): UVM2 $\left(\lambda_{c}=2246 \AA\right)$ magnitude in the AB system; Column (7): UVW1 $\left(\lambda_{c}=2600 \AA\right)$ magnitude in the AB system; Column (8): $\mathrm{U}\left(\lambda_{c}=3465 \AA\right)$ magnitude in the $\mathrm{AB}$ system; Column (9): $\mathrm{B}\left(\lambda_{c}=4392 \AA\right)$ magnitude in the $\mathrm{AB}$ system; Column (10): $\mathrm{V}$ $\left(\lambda_{c}=5468 \AA\right)$ magnitude in the $\mathrm{AB}$ system. ${ }^{\mathrm{a}}$ For all non-detections, the corresponding $3 \sigma$ upper limits are given.

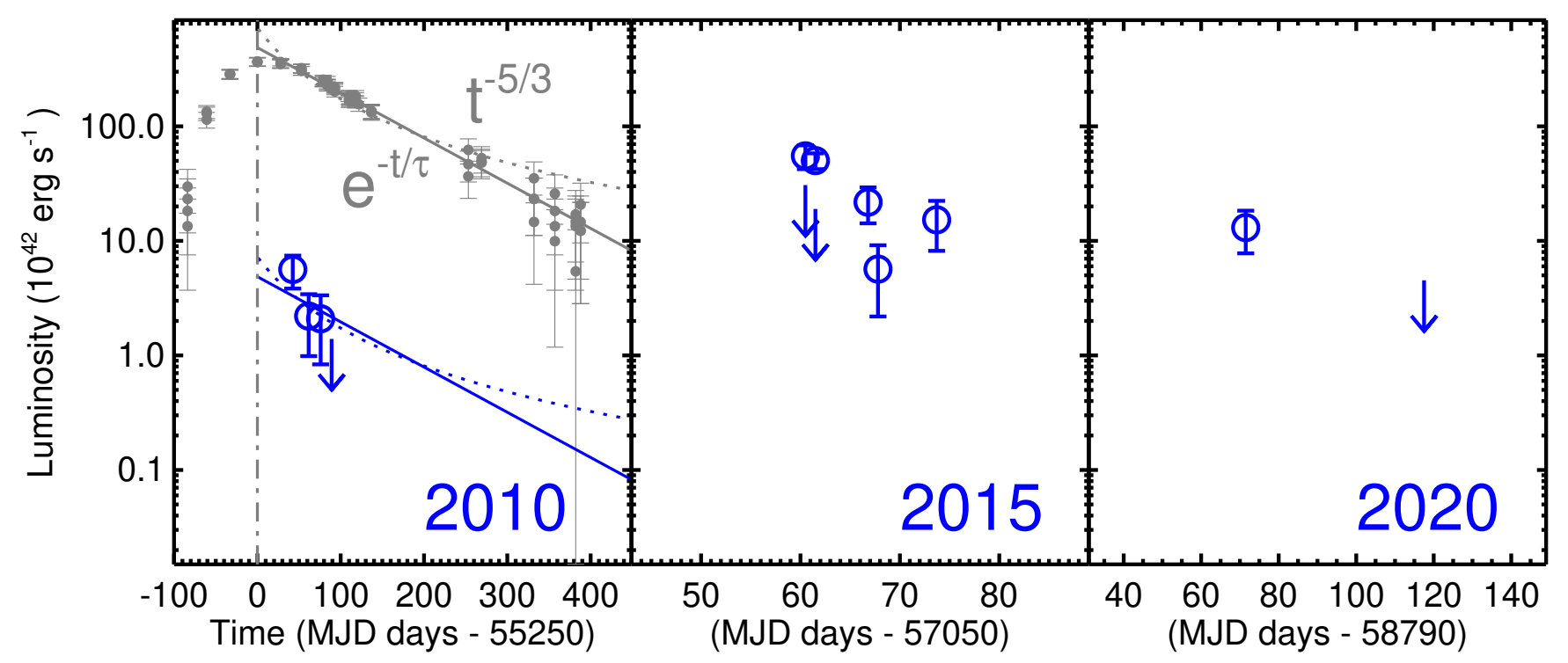

Fig. 1. X-ray light curves observed at different epochs. In the left panel, the evolution of host-subtracted optical luminosity at the V-band is also shown (gray points, in units of $10^{42} \mathrm{erg} \mathrm{s}^{-1}$ ). The solid line shows the best exponential fit, while the dotted line is the fit with the canonical $t^{-5 / 3}$ decay law. The two best-fit lines are scaled downward by a factor of 100 to match the X-ray light curve.

tions, including date, seeing, exposure, spectral resolution and spectral extraction aperture at each epoch are listed in Table 3.

\section{Analysis and results}

\subsection{X-ray variability}

As we mentioned earlier, D11 reported the X-ray detection from observations in 2010, but did not give the details of X-ray properties. We will show that the source is clearly not detected in the last one of the four observations in 2010, indicating a significant flux decay. Here we present a detailed and uniform analysis of all archival X-ray observations, especially for those that are not given in D11, aiming to further investigate the X-ray flux and spectral evolution on longer timescales.
Figure 1 (left) plots the evolution of X-ray luminosity in the 0.3-2 keV for the observations taken on 2010, and $3 \sigma$ upper limit is shown for the non-detection 1 The X-ray flux was converted from count rate using the best-fitting powerlaw model to the stacked spectrum (Section 3.2). It is evident that the source brightness declined by a factor of $\gtrsim 3$ over a period of $\sim 50$ days, from $5.6 \pm 1.8 \times 10^{42} \mathrm{erg} \mathrm{s}^{-1}$ to the upper limit of $9 \times 10^{41} \mathrm{erg} \mathrm{s}^{-1}$. Such an evolution of X-ray brightness is not uncommon if due to a TDE (Blanchard et al. 2017), but no conclusive evidence can be drawn as X-ray variability by even larger factors is often observed in NSy1s. To gain further insight into the nature of X-ray emission, we present the evolution of host-subtracted optical lu-

\footnotetext{
1 Since the $1 \sigma$ detection from the background fluctuation results in less than one net count, we estimated conservatively the upper limit by requiring the detection of one net count, as did in Shu et al. (2020).
} 
minosity at the V-band during the period of outburst (gray dots). We estimate the host galaxy flux from the plateau of light curve before the optical outburst, which is $17.51 \pm 0.1 \mathrm{mag}$ and consistent with that estimated by D11. Note that we used the post-DR2 data from the CRTS survey to construct the host-subtracted light curve (Graham et al. 2017), so it is slightly better sampled than that presented in D11 where only data up to $\sim 300$ days after outburst are shown. While the luminosity evolution can be well described by an exponential law $L=L_{0} e^{-\left(t-t_{0}\right) / \tau}$, the fit with the popular $t^{-5 / 3}$ decay law is worse, especially for the data after $\sim 150$ days since the peak. Although the X-ray luminosity appears to follow the similar evolution (blue curves), it is nearly two orders of magnitude lower than the optical luminosity at the same epochs. We will discuss possible reasons for the relative faintness of the X-ray emission in Section 4.2.

Although the source becomes invisible in the last observation performed on 2010, Swift detected it again in 2015 in $\mathrm{X}$-rays (Figure 1, middle). The highest luminosity recorded is $5.5 \pm 1.3 \times 10^{43} \mathrm{erg} \mathrm{s}^{-1}$, corresponding to a rebrightening by a factor of $>30$ relative to the previous non-detection. In addition, the source declines in flux quickly by a factor of $\sim 4-10$ over two weeks. A previous study of CSS1029 by stacking individual observations claimed the detection of X-ray emission in 2015 as the same order of magnitude as the first observation in 2010 (Auchettl et al.2017). However, this is not true when considering the flux decay and particularly the difference between the lowest state in 2010 and highest state in 2015, which are not considered in past analyses. While the source is detected in the first observation performed on Jan 2020 with $\sim 6 \pm 2$ net counts, it becomes invisible again in the second observation about a month later (Figure 1, right). Although we lack enough X-ray observations to monitor the luminosity evolution since 2015 , the above analysis suggests that the long-term X-ray variability is presented in CSS1029, by factors of $\sim 10$ on time-scales as short as a few tens of days, consistent with that seen in NSyls.

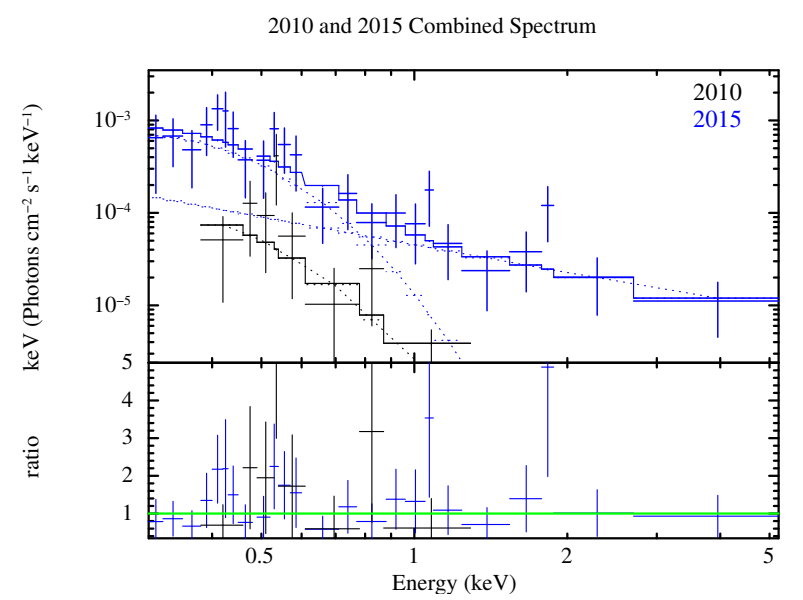

Fig. 2. The X-ray spectrum by stacking three observations in 2010 (black) and five observations in 2015 (blue). The dotted line shows the best-fitted model consisting of a powerlaw and a blackbody component. Note that the spectral fittings were performed jointly to the 2010 and 2015 data based on this model. The corresponding data to model ratios are shown in bottom panel.

\subsection{X-ray spectral analysis}

Because of short exposures, the spectral signal-to-noise ratios (S/Ns) for most of the individual Swift observations are not suf-
Table 2. Spectral fitting results for the Swift 2010 and 2015 data.

\begin{tabular}{ccccc}
\hline \hline Model & $\begin{array}{c}N_{\mathrm{H}} \\
\left(10^{21} \mathrm{~cm}^{-2}\right)\end{array}$ & $\Gamma$ & $\begin{array}{c}k T \\
(\mathrm{eV})\end{array}$ & $C /$ dof \\
\hline \multicolumn{5}{c}{ Swift 2010} \\
\hline powerlaw & $<5.6$ & $3.89_{-1.94}^{+2.75}$ & $126_{-57}^{+90}$ & $7.2 / 7$ \\
\hline blackbody & $0.12^{f}$ & & \\
\hline powerlaw & $<1.0$ & $3.36_{-0.36}^{+0.38}$ & $36.4 / 40$ \\
powerlaw+bbody & $0.12^{f}$ & $2.16_{-1.22}^{+1.02}$ & $101 \pm 28$ & $33.3 / 39$ \\
\hline \hline
\end{tabular}

Notes. ${ }^{f}$ The parameter is fixed in the spectral fittings.

ficient to perform meaningful fittings. Thus, in order to obtain a spectrum with better $\mathrm{S} / \mathrm{N}$, we combined the spectra from individual observations using the FTOOLS task addascaspec. We added spectral files separately from the three Swift observations taken in 2010 (Swift 2010), and five observations in 2015 (Swift 2015). Non-detections are excluded in the spectral stacking (Table 1). We obtained $17 \pm 5$ and $82 \pm 9$ background subtracted counts in the $0.3-5 \mathrm{keV}$ for the Swift 2010 and 2015, respectively. Since only $6 \pm 3$ net counts are detected in the first Swift observation performed on 2020, we do not consider the 2020 data in the following spectral analysis. The Swift 2010 spectrum appears soft, with most of the counts falling below $1.5 \mathrm{keV}(\sim 80 \%)$. For the Swift 2015 observations, we detected 11 net counts at energies above $1.5 \mathrm{keV}$, indicating the presence of harder X-ray emission (Figure 2). In the following spectral analysis, we grouped the data to have at least 2 counts in each bin to ensure the use of $C$-statistic for the spectral fittings. The Galactic column density was considered and fixed at $N_{\mathrm{H}}^{\mathrm{Gal}}=1.23 \times 10^{20} \mathrm{~cm}^{-2}$. All statistical errors given hereafter correspond to $\Delta C=2.706$ for one interesting parameter, unless stated otherwise.

As a first step, we performed the spectral fittings to the combined Swift 2015 spectrum which has the best spectral quality, using a simple absorbed powerlaw model. The fitting result is acceptable, with a powerlaw index $\Gamma=3.36_{-0.34}^{+0.36}$ and $C / d o f=36.4 / 40$. The intrinsic absorption is not required, with an upper limit on the column density of $N_{\mathrm{H}}<1 \times 10^{21} \mathrm{~cm}^{-2}$. Although loosely constrained, the power-law component is relatively steep among AGNs (Grupe et al. 2010). Thus we attempted to fit the spectrum with a blackbody (zbbody in XSPEC), but found an excess of emission at energies above $\sim 1.5 \mathrm{keV}$. The addition of a powerlaw to the model improves the fit significantly ( $C$-value is decreased by 24.9 for two extra parameters). In this case, we obtain a blackbody temperature of $k T_{\mathrm{BB}}=101 \pm 28 \mathrm{eV}$, and the powerlaw component becomes flatter with $\Gamma=2.16_{-1.22}^{+1.02}$, which are consistent with the typical values observed in NSyls.

Similar spectral analysis was also performed for the Swift 2010 data, which can be described by a simple absorbed powerlaw model with photon index $\Gamma=3.89_{-1.94}^{+2.75}$. The photon index is comparable to that observed in TDEs which can be ascribed to the thermal disk emission at soft X-ray bands (Komossa 2015). We found a single blackbody model providing an equally good fit to the Swift 2010 data, without requirement for an additional powerlaw component. Since the spectral quality is poor for the 2010 data, we cannot tell whether the source is intrinsically weak at hard X-rays or such a component is simply eluded detection due to insufficient exposure. Given that the $0.3-1 \mathrm{keV}$ spectral shape is consistent with each other between the 2010 and 2015 

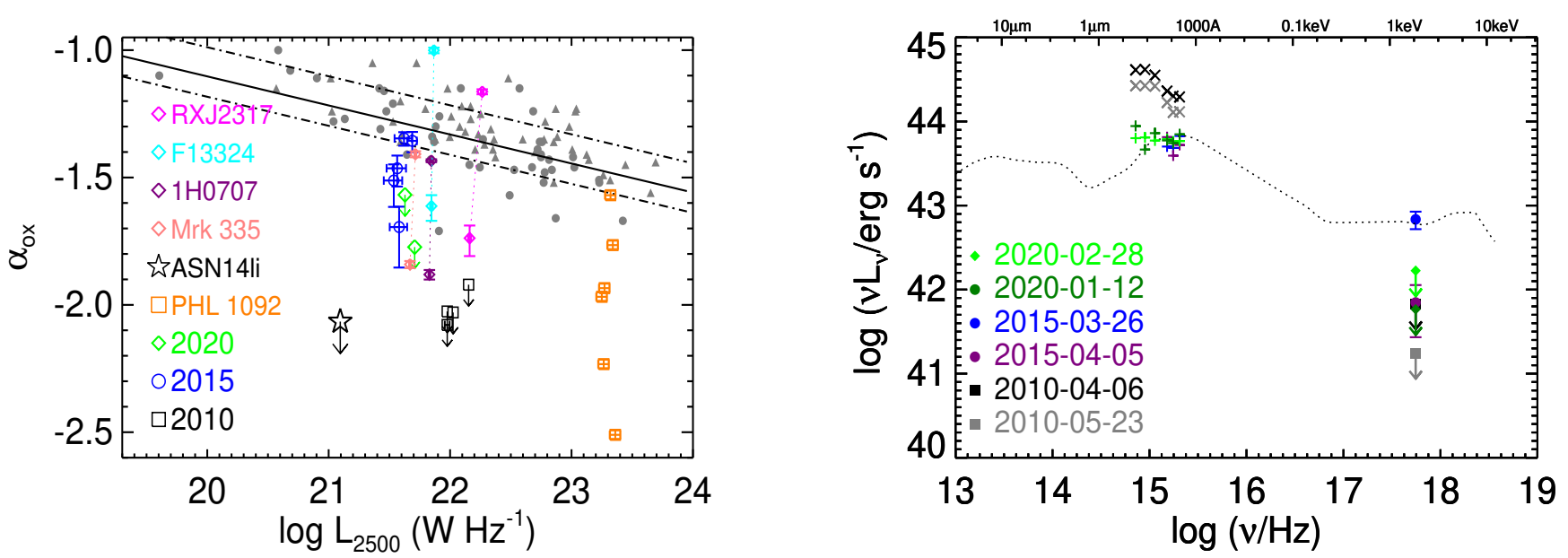

Fig. 3. Left: UV-to-X-ray spectral index $\alpha_{\text {ox }}$ versus $2500 \AA$ luminosity for CSS1029 (black, green and blue symbols). The solid line represents the fit to the X-ray selected AGNs from Grupe et al. (2010). The NSy1s are shown in gray dots, while the broad-line Sy1s are shown in gray triangles. For comparison, we also plot the $\alpha_{\mathrm{ox}}$ for four typical NSy1s with extreme X-ray variations. The $\alpha_{\mathrm{ox}}$ were calculated in the same manner as CSS1029. For clarity, only the two data points representing observations between the high and low sate are shown. The open star represents the data for the thermal TDE ASASSN-14li in the flare state (van Velzen et al. 2016). Right: Optical to X-ray SED of CSS1029. For clarity, only the data for the highest and lowest state within each epoch are shown. The median SED from radio quiet quasars (Elvis et al. 1994) is also shown for comparison (dotted line), for which the luminosity is scaled to that of UVW2 obtained in 2015 high state.

observations, we then considered a joint fit to the two data sets in the $0.3-5 \mathrm{keV}$. We used the best-fitting model for the 2015 spectrum, comprising a thermal blackbody model plus a power law, modified by the Galactic absorption. All the parameters are fixed to be the same except for flux normalizations. We obtained an acceptable joint fit $(C / d o f=43.2 / 48)$ with $k T \sim 101 \mathrm{eV}$ and $\Gamma \sim 2.04$. Based on this model, we found an $0.3-2 \mathrm{keV}$ flux of $7.02 \times 10^{-14} \mathrm{erg} \mathrm{cm}^{-2} \mathrm{~s}^{-1}$ and $5.42 \times 10^{-13} \mathrm{erg} \mathrm{cm}^{-2} \mathrm{~s}^{-1}$ , and $2-10 \mathrm{keV}$ flux of $1.98 \times 10^{-14} \mathrm{erg} \mathrm{cm}^{-2} \mathrm{~s}^{-1}$ and $1.53 \times$ $10^{-13} \mathrm{erg} \mathrm{cm}^{-2} \mathrm{~s}^{-1}$ for the 2010 and 2015 data, respectively. Note that the hard X-rays are clearly not detected for the 2010 observations, hence the above $2-10 \mathrm{keV}$ flux from the model extrapolation would serve as only an upper limit.

\subsection{UV emission and optical-to-X-ray spectral energy distribution}

As we shown above, the X-ray emission appears relatively faint during the period of optical outburst in CSS1029. In order to gain insights into the X-ray properties, we turn now to the analysis of the UV emission and its flux ratio to X-rays. All the Swift observations provide simultaneous UV photometric data through UVOT in the filter UVW2 $\left(\lambda_{c}=1928 \AA\right), \operatorname{UVM} 2\left(\lambda_{c}=2246 \AA\right)$ and UVW1 $\left(\lambda_{c}=2600 \AA\right)$, respectively. For the observations performed on 2010 and 2020, the simultaneous optical data are also available (Table 1). It is interesting to note that while the X-ray flux increases by a factor of up to $>30$ between 2010 and 2015 observations, the UV variations, however, have much smaller amplitudes. For example, the maximum UV variation for the UVW2 is $\sim 1.54 \mathrm{mag}$, corresponding to only a factor of $\sim 4$ drop in the monochromatic flux between the two epochs. The UV variations are neither strongly correlated with the X-ray ones within each epoch.

The availability of simultaneous UV and X-ray data allows us to derive reliably the UV-to-X-ray slope for each observation. The UV-to-X-ray slope is often defined by monochromatic flux ratio between $2 \mathrm{keV}$ and $2500 \AA$ in the rest frame, parameterized with $\alpha_{\text {ox }}=0.384 \log \left(f_{2 \mathrm{keV}} / f_{2500 \AA}\right)$. To calculate the $2500 \AA$ flux, we used the observed flux at the bluest UV band $(\sim 1928 \AA$, UVW2), which is expected less affected by host contamination. Then the UVW2 flux was extrapolated to the $2500 \AA$ by assuming a typical UV spectral index of $\alpha=0.65\left(S_{v} \propto v^{-\alpha}\right.$, Grupe et al. 2010). We note that adopting a different slope of $\alpha$ between 1 and 0 in the extrapolation would result a change by $\lesssim 50 \%$ in the $2500 \AA$ flux and hence by $\lesssim 20 \%$ in the $\alpha_{\text {ox }}$. The X-ray flux density at $2 \mathrm{keV}$ was estimated by the best-fitting model that was used to perform the joint fit to the 2010 and 2015 data, and corrected for the Galactic absorption (Section 3.2). The model consists of a powerlaw with $\Gamma=2.04$ and a blackbody component with $k T=101 \mathrm{eV}$. In order to avoid introducing modeldependent biases, we adopted the same model to estimate the 2 $\mathrm{keV}$ flux for the Swift 2020 data. Since no X-rays above $1.5 \mathrm{keV}$ are detected for the 2010 and 2020 observations, we considered all the $\alpha_{\text {ox }}$ as upper limits for these epochs. Our measurements imply that CSS1029 has a much steeper UV to X-ray spectral slope $\left(\alpha_{\text {ox }} \lesssim-2\right)$ in 2010 than that in $2015\left(\alpha_{\text {ox }} \simeq-1.5\right)$.

It is well known that the $\alpha_{\text {ox }}$ are correlated with the $2500 \AA$ luminosity for AGNs, which are increasingly X-ray weak for higher UV luminosities (e.g., Steffen et al. 2006). Such a relation for the X-ray selected AGNs is shown by the solid line (Grupe et al. 2010) in Figure 3 (left), and the dotted line represents the approximately $1 \sigma$ scatter of the distribution. The NSy1s from the sample are shown in gray filled circles, while the broad-line Sy1s are shown in triangles. It can be seen that the $\alpha_{\text {ox }} \simeq-1.35$ at the highest state (rebrightening stage in 2015) is quiet typical for CSS1029 as a normal NSy1. However, the source became increasingly X-ray weak in the subsequent observations, down to an X-ray level comparable to that obtained with the most recent observations in 2020, falling significantly below the extrapolation of the $\alpha_{\mathrm{ox}}-L_{2500 \AA}$ relation. The average $\Delta \alpha_{\text {ox }}=\alpha_{\text {ox }}-\alpha_{\text {ox,exp }}=-0.191$ and $<-0.379$ for the 2015 
and $2020 \mathrm{dat}^{2}$, corresponds to be X-ray weaker than $\alpha_{\text {ox,exp }}$ at a $2.38 \sigma$ and $4.74 \sigma$ level, respectively. In comparison with the 2015 and 2020 data, CSS1029 is even more (hard) X-ray weak in 2010 according to the $\Delta \alpha_{\text {ox }}$. Such an extreme X-ray weakness is unusual among AGNs, when compared with the NSy1s in an extreme X-ray faint state, such as Mrk 335 (Grupe et al. 2012b), 1H0707 (Fabian et al. 2012), IRAS F13324-3809 (Buisson et al. 2018) and RX J2317.8-4422 (Grupe et al. 2019). This is similar to the X-ray weak quasar phenomenon, such as PHL 1092 from XMM-Newton monitoring observations (Miniutti et al. 2012), suggesting CSS1029 might be one of AGNs with extreme $\alpha_{\text {ox }}$ variability ever found. Alternatively, such an X-ray weakness might be expected if the X-ray emission was dominated by a soft blackbody component as originated from the transient disk accretion in the process of TDE. In fact, the ultrasoft X-ray spectra have been found common in TDEs (Komossa 2015; Auchettl et al. 2017). We also plot the $\alpha_{\text {ox }}$ (open star in Figure 3 (left)) for the best-studied thermal TDE ASASSN-14li in the flare state (e.g., van Velzen et al.|2016), and find it is comparable to the 2010 data for CSS1029, suggesting a possible connection between the two.

A further perspective on the unusual X-ray properties of CSS1029 comes from the detailed comparison of optical-to-Xray spectral energy distribution (SED) between different epochs, as shown in Figure 3 (right). For clarity, we show only the data from the high and low state for the three epochs. The recent Swift observations in 2020 revealed a similar flux as the one in the last observation of 2015, followed by a drop by a factor of $\geq 3$ within $\sim 50$ days, indicating a possibly new X-ray variability. For comparison, the median SED of radio-quiet quasars, scaled to the UVW2 flux in the 2015 high state, is also shown (dotted line). The UV-to-X-ray SED of CSS1029 in the 2015 high state is consistent with those of typical quasars, while it is X-ray weak by an order of magnitude in the low state, likewise for the 2020 data. We note that the optical-to-X-ray SEDs for observations in 2010 might be different from the median SED of typical quasars, even for the high state data, suggestive of extreme X-ray weakness of intrinsic AGN emission (e.g., Miniutti et al. 2012; Liu et al. 2019). The results are consistent with the above analysis of $\alpha_{\mathrm{ox}}$ variability.

\subsection{Optical spectrum and its evolution}

High-amplitude X-ray flaring has been detected in multiple types of AGNs, some of which are accompanied by a strong change in the flux of the broad Balmer lines (Shappee et al. 2014; Parker et al. 2016, Oknyansky et al. 2019), probably due to an increase in the accretion rate. We performed two follow-up spectroscopy observations with P200 on Feb 2018 (P18) and Feb 2020 (P20), and three with YFOSC on April 2020 (Y20), Jan 2021 (Y21a) and May 2021 (Y21b), respectively. The 2020 observations were carried out promptly after the recent detection of X-rays. Figure 4 (upper panel) shows the follow-up spectra as well as the earlier spectrum from the Sloan Digital Sky Survey (SDSS) for comparison. It appears that spectral variations are present between different epochs. To account for different observing conditions and apertures that may lead to spectral differences, we calibrate the spectra using the observed [O III] $\lambda 5007$ flux, under the assumption that the [O III] narrow-line flux does not vary over the timescale of interest (e.g., Peterson et al. 2013). The calibrated, starlight and continuum-subtracted spectra in the $H_{\alpha}$ and $H_{\beta}$ re-

\footnotetext{
${ }^{2} \alpha_{\text {ox,exp }}$ is the $\alpha_{\text {ox }}$ expected from the Grupe et al. (2010) $\alpha_{\text {ox }}-L_{2500 \AA}$ relation.
}

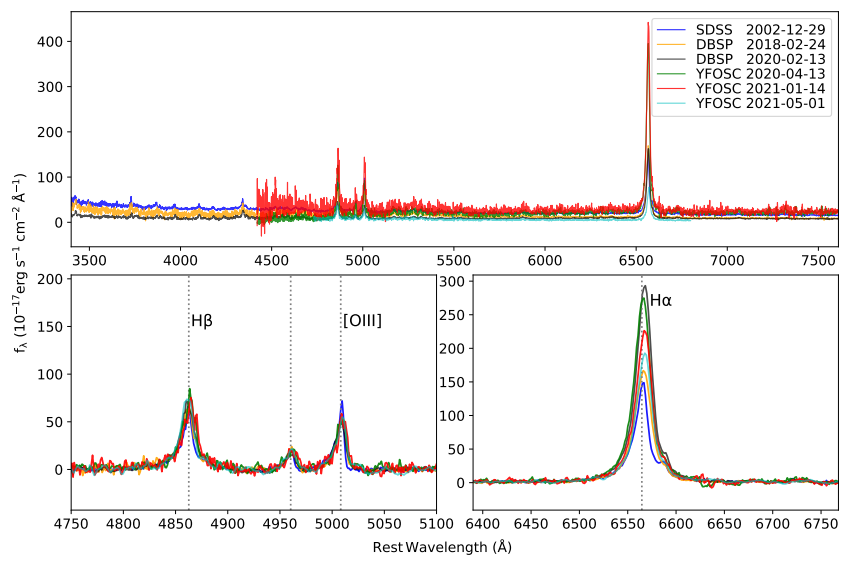

Fig. 4. The P200/DBSP spectrum observed on Feb 2018 (orange) and Feb 2020 (gray), YFOSC spectrum observed on April 2020 (green), Jan 2021 (red) and May 2021 (cyan), in comparison with the archival SDSS spectrum taken on 2002 (blue). The lower panel shows a zoomed-in view of the starlight- and continuum-subtracted spectra in the $H_{\beta}$ and $H_{\alpha}$ region, which are calibrated using the [O $\left.\mathrm{III}\right]$ narrow-line flux.

gion are shown in Figure 4 (lower panel). Significant changes in the $H_{\alpha}$ emission line is obvious, while the same trend is not visible in the $H_{\beta}$ line.

To further explore the $H_{\alpha}$ profile changes, we performed detailed spectral fittings to measure the AGN continuum and the emission lines. Firstly, the Galactic extinction was dereddened using the dust map provided by Schlegel et al. (1998). Then we tried to decompose the spectra into a host galaxy and AGN component with the principal component analysis method (Yip et al. 2004). However, we obtained negative flux for the host galaxy, which means the spectra are entirely dominated by the AGN emission. Therefore, we did not consider the host galaxy component in our following spectral analysis. The continuum was then modeled by a power law plus a third-order polynomial with the region around the broad emission lines masked out, while the optical and UV Fe II were modeled by empirical templates from literature (Boroson \& Green 1992; Vestergaard \& Wilkes 2001). For the Balmer emission lines, Drake et al. (2011) found that there are three significant components with narrow, medium, and broad velocity widths. We hence used three Gaussians to model the $\mathrm{H} \alpha$ (and $\mathrm{H} \beta$ ) line. For [O III] doublets, each narrow line is fitted by two Gaussians, while that of [N II] and [S II] doublets is represented by a single Gaussian. During spectral fittings, the profiles and redshifts of narrow lines are tied. In addition, flux ratios of the [O III] doublets $\lambda 5007, \lambda 4959$ and [N II] $\lambda 6583, \lambda 6548$ are fixed to their theoretical values. Considering the lower $\mathrm{S} / \mathrm{N}$ in the $\mathrm{H} \beta$ region of the $\mathrm{P} 18$ and $\mathrm{Y} 20$ spectrum, the upper limit on the FWHM (Full Width at Half Maximum) of the $\mathrm{H} \beta$ broad component is bounded and set by that of $\mathrm{H} \alpha$. The narrow line components of Balmer lines are assumed to be constant and fixed to that of preflare SDSS spectrum. The median flux between rest-frame 5095 and $5105 \AA$ is taken to calculate the 5100 $\AA$ luminosity. Uncertainties on the spectral parameters, including the $5100 \AA$ luminosity, were derived using Monte Carlo simulations, where the observed spectrum was randomly perturbed by adding Gaussian noise at each wavelength bin, with amplitude determined by the flux error. This was repeated 100 times, resulting in a distribution of the best-fitting parameter of interest, the standard deviation of which was considered as its $1 \sigma$ error.

In Figure 5, we present the spectral decompositions of $\mathrm{H} \alpha$ line observed in six epochs. The emission-line spectral fitting 
Table 3. Spectroscopic observations and emission line fitting results.

\begin{tabular}{|c|c|c|c|c|c|c|}
\hline Obs. $\log$ & SDSS & P200 & P200 & YFOSC & YFOSC & YFOSC \\
\hline Obs. date & 2002 Dec & $2018 \mathrm{Feb}$ & $2020 \mathrm{Feb}$ & 2020 April & 2021 Jan & 2021 May \\
\hline Seeing $(\operatorname{arcsec})$ & $1.78-2.2$ & 3.3 & 1.5 & 2.1 & 1.7 & 2.1 \\
\hline Aperture width (arcsec) & $3^{\dagger}$ & 1.5 & 1.5 & 1.5 & 1.5 & 1.5 \\
\hline Spectral resolution $(\lambda / \Delta \lambda)^{\ddagger}$ & 1780 & 1080 & 1080 & 380 & 380 & 810 \\
\hline Exposure (second) & 3000 & 2700 & 800 & 1800 & 1800 & 2400 \\
\hline \multicolumn{7}{|l|}{ Parameters } \\
\hline $\mathrm{H}_{\alpha \mathrm{b}}\left(10^{-14} \mathrm{erg} \mathrm{cm}^{-2} \mathrm{~s}^{-1}\right)$ & $0.85 \pm 0.60$ & $1.25 \pm 0.1$ & $2.8 \pm 0.09$ & $2.6 \pm 0.08$ & $2.0 \pm 0.23$ & $1.7 \pm 0.01$ \\
\hline $\mathrm{H}_{\alpha \mathrm{m}}\left(10^{-14} \mathrm{erg} \mathrm{cm}^{-2} \mathrm{~s}^{-1}\right)$ & $1.42 \pm 0.07$ & $2.76 \pm 0.1$ & $4.1 \pm 0.1$ & $4.1 \pm 0.09$ & $3.4 \pm 0.27$ & $2.9 \pm 0.01$ \\
\hline $\mathrm{H}_{\alpha \mathrm{n}}\left(10^{-14} \mathrm{erg} \mathrm{cm}^{-2} \mathrm{~s}^{-1}\right)$ & $0.39 \pm 0.04$ & $0.39 \pm 0.01$ & $0.39 \pm 0.01$ & $0.39 \pm 0.01$ & $0.39 \pm 0.01$ & $0.39 \pm 0.01$ \\
\hline FWHM $\left[\mathrm{H}_{\alpha \mathrm{b}}\right]\left(\mathrm{km} \mathrm{s}^{-1}\right)$ & $2860 \pm 170$ & $3380 \pm 512$ & $2653 \pm 111$ & $2790 \pm 92$ & $3046 \pm 236$ & $3476 \pm 32$ \\
\hline $\mathrm{FWHM}\left[\mathrm{H}_{\alpha \mathrm{m}}\right]\left(\mathrm{km} \mathrm{s}^{-1}\right)$ & $807 \pm 38$ & $1034 \pm 32$ & $846 \pm 13$ & $892 \pm 11$ & $911 \pm 21$ & $920 \pm 2.5$ \\
\hline FWHM $\left[\mathrm{H}_{\alpha \mathrm{n}}\right]\left(\mathrm{km} \mathrm{s}^{-1}\right)$ & $318 \pm 15$ & $318^{f}$ & $318^{f}$ & $318^{f}$ & $318^{f}$ & $318^{f}$ \\
\hline $\mathrm{H}_{\beta \mathrm{b}}\left(10^{-15} \mathrm{erg} \mathrm{cm}^{-2} \mathrm{~s}^{-1}\right)$ & $6.7 \pm 0.3$ & $7.8 \pm 1.6$ & $7.5 \pm 0.9$ & $7.4 \pm 0.6$ & $7.9 \pm 2.0$ & $7.4 \pm 0.2$ \\
\hline $\mathrm{H}_{\beta \mathrm{m}}\left(10^{-15} \mathrm{erg} \mathrm{cm}^{-2} \mathrm{~s}^{-1}\right)$ & $3.2 \pm 0.4$ & $4.9 \pm 1.6$ & $5.6 \pm 0.9$ & $6.3 \pm 0.5$ & $5.5 \pm 2.2$ & $5.5 \pm 0.3$ \\
\hline $\mathrm{H}_{\beta \mathrm{n}}\left(10^{-15} \mathrm{erg} \mathrm{cm}^{-2} \mathrm{~s}^{-1}\right)$ & $1.3 \pm 0.3$ & $1.3 \pm 0.1$ & $1.3 \pm 0.08$ & $1.3 \pm 0.01$ & $1.3 \pm 0.01$ & $1.3 \pm 0.01$ \\
\hline $\mathrm{FWHM}\left[\mathrm{H}_{\beta \mathrm{b}}\right]\left(\mathrm{km} \mathrm{s}^{-1}\right)$ & $2721 \pm 154$ & $2821 \pm 625$ & $2449 \pm 213$ & $2787 \pm 36$ & $2562 \pm 222$ & $2099 \pm 87$ \\
\hline $\mathrm{FWHM}\left[\mathrm{H}_{\beta \mathrm{m}}\right]\left(\mathrm{km} \mathrm{s}^{-1}\right)$ & $736 \pm 67$ & $746 \pm 88$ & $920 \pm 86$ & $860 \pm 62$ & $866 \pm 229$ & $650 \pm 24$ \\
\hline $\mathrm{FWHM}\left[\mathrm{H}_{\beta \mathrm{n}}\right]\left(\mathrm{km} \mathrm{s}^{-1}\right)$ & $318 \pm 15$ & $318^{f}$ & $318^{f}$ & $318^{f}$ & $318^{f}$ & $318^{f}$ \\
\hline$L_{5100 \AA}\left(10^{43} \mathrm{erg} \mathrm{s}^{-1}\right)$ & $6.6 \pm 1.0$ & $4.2 \pm 1.0$ & $4.05 \pm 1.0$ & $3.7 \pm 1.0$ & $3.8 \pm 1.0$ & $4.9 \pm 1.0$ \\
\hline
\end{tabular}

Notes. ${ }^{\dagger}$ It corresponds to the fiber size to extract the SDSS spectroscopy, which has a diameter of $3^{\prime \prime} .{ }^{\ddagger}$ The spectral resolution at $\lambda=6600 \AA$. ${ }^{f}$ The parameter is fixed at the best-fit value from the spectral decomposition of the pre-flare SDSS data.
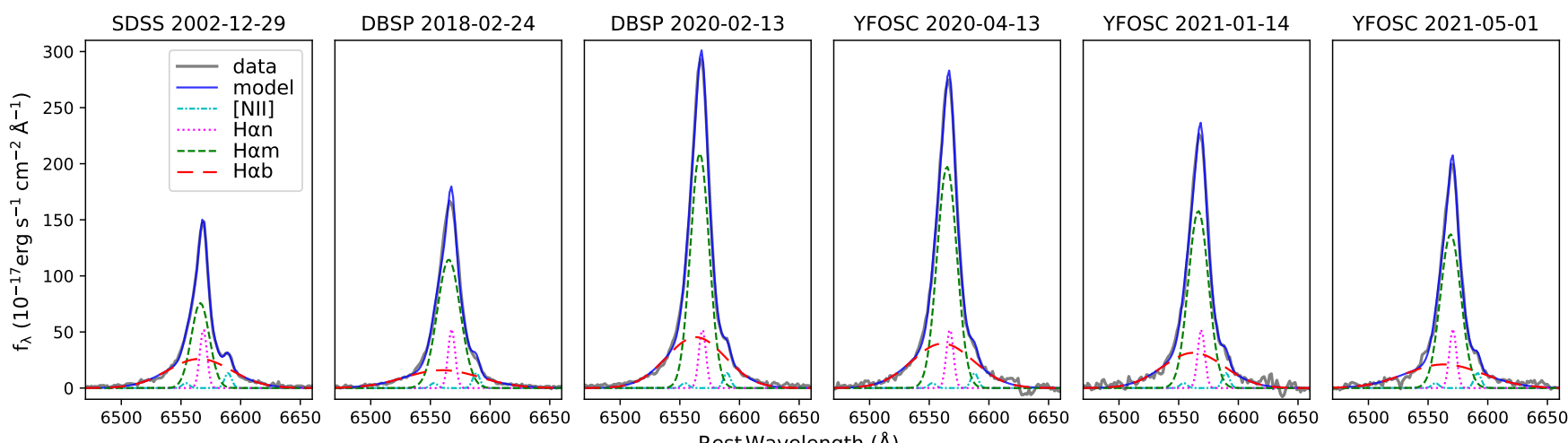

Fig. 5. Illustration of the emission line profile fitting in the $H_{\alpha}+[\mathrm{N}$ II] region observed at different epochs.

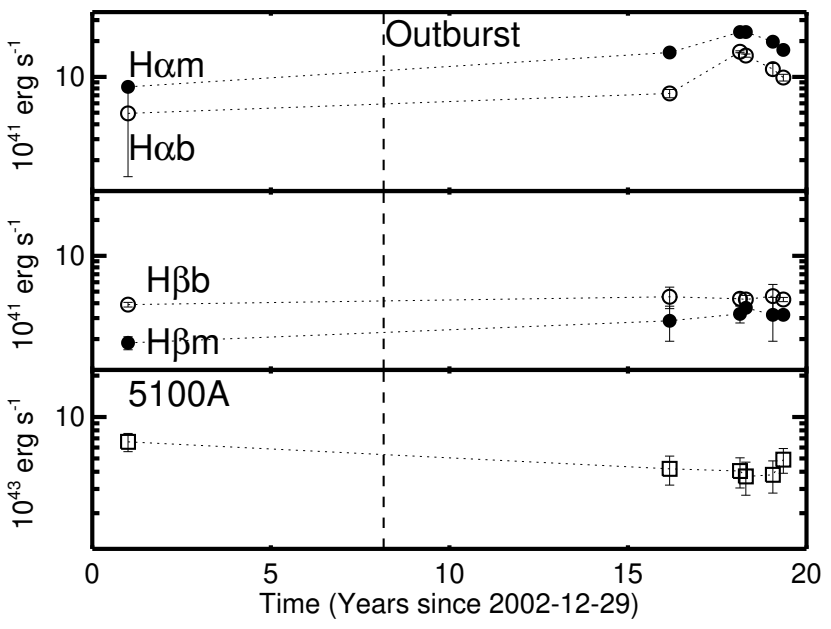

Fig. 6. Variation of $\mathrm{H} \alpha, \mathrm{H} \beta$ and the continuum luminosity at $5100 \AA$ as a function of time. Both broad and intermediate emission line components are shown. The dashed vertical line indicates the time of peak luminosity of optical outburst observed on 2010 Feb 23 (D11). results are shown in Table 3 . Interestingly, we found that the broad and intermediate component of $H_{\alpha}$ emission line observed with P200 on 2018 are showing slight increase in flux with respect to the SDSS ones, and the fluxes are rising to a peak during the P200/DBSP observation on 2020, by a factor of $\sim 3.3$ and $\sim 2.9$ than that of SDSS ones (Table 3). Although the actual time for the onset of $\mathrm{H} \alpha$ strengthening is not known, it remains strong at least for $\sim 2$ months based on the current data. Our recent YFOSC spectrum taken on Jan 2021 and May 2021 shows that the broad $\mathrm{H} \alpha$ flux appears to have declined, though it is still a factor of $\sim 2$ higher than that of pre-flare SDSS spectrum. This strongly suggests that the $\mathrm{H} \alpha$ brightening is a transient phenomenon. We argue that such an $\mathrm{H} \alpha$ flux variation is not due to observational effects, as our simulations (see the details in Appendix A) have demonstrated that the changes in the $\mathrm{H} \alpha \mathrm{EW}$ is at most at a level of $7 \%$ under the conditions of five spectroscopic observations (Table 3), i.e., for the seeing between $1.5^{\prime \prime}$ and 3.3", and the slit width between $1.5^{\prime \prime}$ and $2.5^{\prime \prime}$. Timely spectroscopy monitoring observations are highly encouraged to determine how long will the $\mathrm{H} \alpha$ brightening last. 
Since the optical broad emission lines (BELs) are believed to arise largely from the high-density gas clouds in BLR (Broad Line Region) that is photoionized by an intense central continuum radiation, we would naively expect correlated changes in the BEL strengths with the intrinsic variations in the incident ionizing continuum flux. This unique property between the continuum and emission line variations constitutes the foundation of the reverberation mapping technique (RM, see Blandford \& McKee 1982), and has proven observationally extremely useful to probe the spatial distribution and kinematics of the BEL gas (see Peterson 1993, and references therein). Greene \& Ho (2005) found that the luminosity of $\mathrm{H} \alpha$ BEL is correlated with the continuum luminosity at $5100 \AA$ for an ensemble of AGNs. The best-fit relation suggests $L_{H \alpha} \propto L_{5100 \AA}^{1.157}$, with an rms scatter of $\sim 0.2$ dex (Greene \& Ho 2005). If assuming CSS1029 follows this relation, the observed enhancement in the $\mathrm{H} \alpha$ flux (combined broad and intermediate components) on 2020 by a factor of $\sim 1.8$ in CSS1029 with respect to that of 2018 would indicate a corresponding increase in the $5100 \AA$ continuum flux by $\sim 0.6 \pm 0.5 \mathrm{mag}$. The actual $5100 \AA$ luminosity appears, however, to remain little changes with time during our follow-up spectroscopy observing campaign, as shown in Table 3 and Figure 6. Such a reverse evolution between the $\mathrm{H} \alpha$ line and continuum variations is even extreme if compared with the pre-flare SDSS spectrum.

Strictly speaking, the above comparison is unfair because the flux responsivity of $\mathrm{H} \alpha$ BELs to the continuum variations in individual AGNs might be different from the above luminosityluminosity relation, such as AGNs with RM observations for which the line responsivity typically has a scatter of less than 0.15 dex (e.g., Shapovalova et al. 2012, 2019). To take into account this effect, we compare in Figure 7 the variability of $\mathrm{H} \alpha$ BELs with the continuum variability of CSS1029 at $g$-band observed by ZTF, which has the best sampling in light curve covering our follow-up spectroscopic observations (thick vertical lines). For clarity, the $\mathrm{H} \alpha$ light curves have been normalized to the mean value of $g$-band flux at the date of our P18 observation. It can be seen that while the $g$-band flux remains constant with little evolution with time, the variability amplitudes for $\mathrm{H} \alpha$ BELs are much larger, above the $3 \sigma$ upper limit on the $g$-band variability. Under the assumption of $F_{\text {line }} \propto F_{\text {cont }}^{\eta}$ in the RM model, the result clearly suggests that the $\mathrm{H} \alpha$ BEL variability in CSS1029 is not relevant to the continuum variation.

\subsection{Multi-wavelength photometric light curves}

CSS1029 was discovered by the CRTS survey on 2010 Feb (D11), but only optical photometry up to $\sim 300$ days since outburst is shown. We retrieved the optical V-band photometric data from the CRTS websit ${ }^{3}$ and updated to match the long-term light curve as presented in Graham et al. (2017). The V-band light curve is displayed in Figure 8 . It can be seen that the source clearly displays two distinct states in the optical band: a long quiescent state and an outburst state which differs in brightness by $\sim 1.5-1.8 \mathrm{mag}$. The quiescent brightness decreases by $\sim 0.3 \mathrm{mag}$ after the outburst, suggesting a slight change in the accretion rate. In addition, we built MIR light curves by collecting photometric data at $3.4 \mu \mathrm{m}(\mathrm{W} 1)$ and $4.6 \mu \mathrm{m}$ (W2) from the WISE (Wide-field Infrared Survey Explorer) survey up to Dec 2020. Details on the WISE photometry and lightcurve construction are given in Jiang et al. (2016, 2019). The MIR flares are clearly

\footnotetext{
${ }^{3}$ http://nesssi.cacr.caltech.edu/DataRelease
}

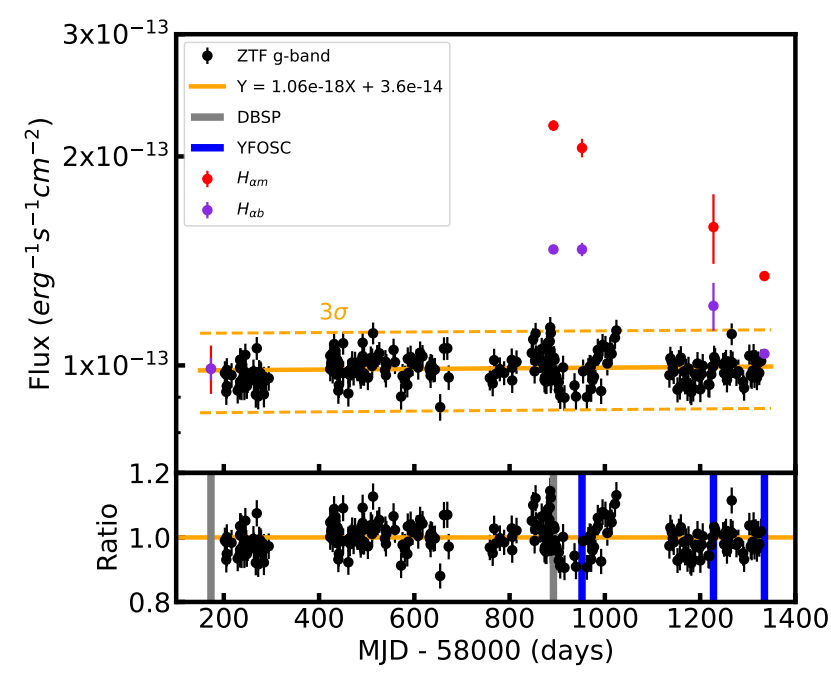

Fig. 7. Comparison between the variation in $\mathrm{H} \alpha$ broad line (purple) and that of medium width component (red) and the observed $g$-band continuum light curve (black points, see also in Figure 8). The orange solid line represents the linear fit, suggesting that the $g$-band flux variability can be described by a constant, with little evolution with time. The dashed line indicates the $3 \sigma$ scatter of flux variability relative to its mean. For clarity, the $\mathrm{H} \alpha$ flux has been normalized to the mean $g$-band flux on the date of Feb 24, 2018, the start of our follow-up spectroscopic observations (thick vertical lines). Bottom panel shows the ratios of the $g$-band fluxes to the best-fit mean.

presented soon after the optical outburst (MJD 55325), probably due to the dust reprocessed emission from the circumnuclear region. The MIR flare is followed by a fading back to a long quiescent level, similar to that observed in the optical. The trend is confirmed with the recent g-band light curve from the ZTF (Zwicky Transient Facility) survey ${ }^{4}$, which displays a plateau over a period of $\sim 3$ years (up to May 2021). We also show the long-term X-ray and UV light curves observed by Swift, though the sampling of the data is much poor.

\subsubsection{Analysis of UV to optical SED during outburst}

We fitted a blackbody model $\left(B_{v}=\frac{2 h v^{3}}{c^{2}} \frac{1}{e^{h v / k T}-1}\right)$ to the UV-tooptical photometric data from the Swift UVOT observations during the period of optical burst, to put constrains on the luminosity, temperature and radius evolution of UV and optical emission of CSS1029. Swift UVOT observations cover a wavelength range from $\sim 1900 \AA$ to $5500 \AA$, which is sensitive to probe the UV-to-optical SED of typical optical TDEs (e.g., van Velzen et al. 2021). In addition, the simultaneous UVOT observations at six filters are helpful to migrate the uncertainties on SED fittings due to the variability in individual bands. We utilized the archival as well as our own target-of-opportunity Swift observations performed on Jan 2020 and Feb 2020 to estimate the quiescent host emission. The host emission at each filter is estimated as the mean of flux between the two epochs, which was then subtracted from the total UVOT measurements to obtain the transient photometry during the period of the outburst (and the errors on host flux were propagated). The transient photometry was then corrected for the Galactic extinction of $E(B-V)=0.015$ mag (Drake et al. 2011). We found that the host-subtracted, Galactic extinction-corrected UV-to-optical SED of CSS1029 can be

\footnotetext{
${ }^{4}$ https://irsa.ipac.caltech.edu/applications/ztf/
} 


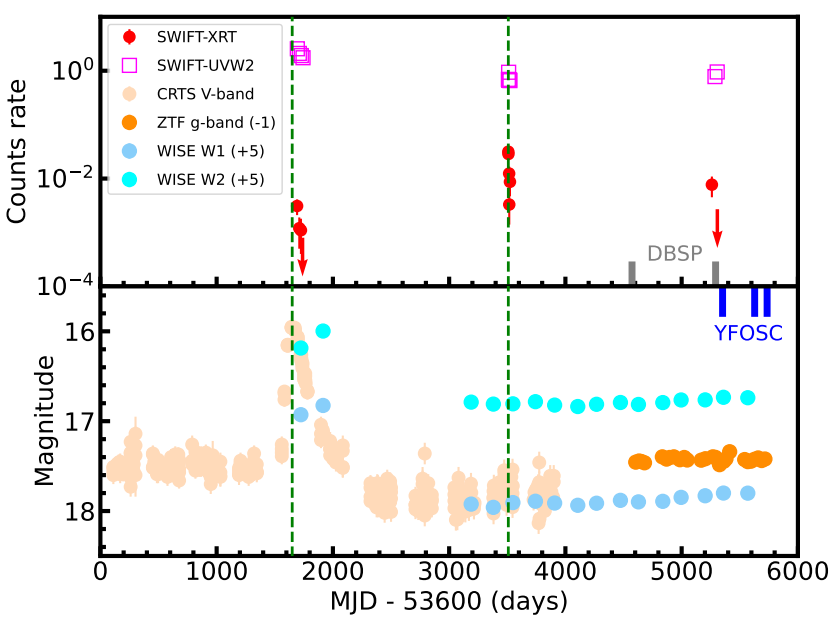

Fig. 8. Multi-wavelength light curves showing the changes in continuum over the past 15 years for CSS1029. Upper panel: The UV (magenta) and X-ray (red) light curves observed from Swift. Bottom panel: The light curve in the optical V-band from the CRTS DR2 (light orange), g-band from the ZTF DR2 (dark orange), and in the MIR $3.4 \mu \mathrm{m}$ (cyan) and $4.6 \mu \mathrm{m}$ (light blue) from WISE. The green dashed line represents the time of optical peak in 2010, and the X-ray peak in 2015, respectively. The gray thick lines show the time of our DBSP spectroscopy observations, while the blue thick lines indicate the time of YFOSC spectroscopy observations.

well described by the blackbody model, with a reduced $\chi^{2} /$ dof of $\sim 0.8-0.9$ (see Appendix B for the fitting results).

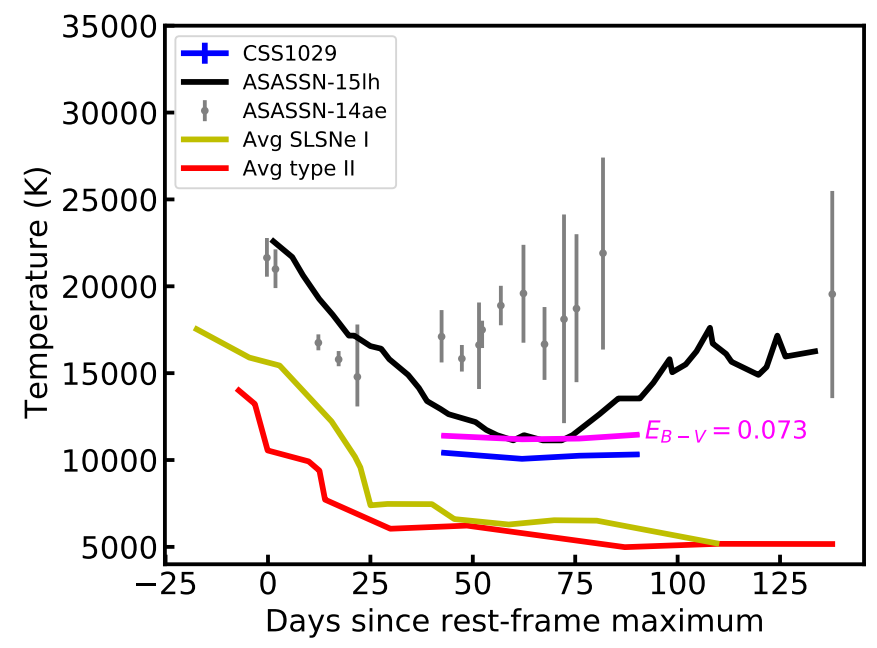

Fig. 9. Upper panel: Temperature evolution of UV-optical emission of CSS1029, as compared with that of superluminous supernovae (SLSNe) and type II SNe (Inserra et al. 2018). The blue curve shows the blackbody temperature measured from the photometric data corrected for the Galactic dust extinction $\left(E_{B-V}=0.015\right)$, while the purple curve represent that corrected for both the Galactic and internal dust extinction $\left(E_{B-V}=0.073\right)$. The latter is estimated from the Balmer decrement of narrow emission lines (Drake et al.2011). The temperature evolution of CSS1029 appears similar to that of optical TDE ASASSN-14ae (gray points, Holoien et al. 2014), as well as the peculiar SLSN ASASSN15lh (black curve, Dong et al. 2016). The latter has been suggested to be more consistent with a TDE based on recent observations (Leloudas et al. 2016).

The SED fitting results indicate a flat evolution of blackbody temperature between $\sim 40-90$ days since the optical peak, with a mean temperature of $\sim 1.03 \times 10^{4} \mathrm{~K}$, as shown in the blue curve in Figure 9 (upper panel). We note that the temperature fit to UV-to-optical SED is very sensitive to internal extinction. Although Drake et al. (2011) suggest that the host galaxy extinction of CSS1029 is a small effect, we estimated a potential internal extinction with the narrow line Balmer decrement as measured from the preflare SDSS spectrum (Drake et al. 2011). By summing the Galactic extinction, we found a total extinction $E_{B-V}=0.073$ assuming an "average" reddening for the diffuse interstellar medium of $R(V)=3.1$. Correcting for the total extinction to the UV-optical SED observed by Swift, the best-fit temperature increases to $\sim 1.13 \times 10^{4} \mathrm{~K}$ (purple curve in Figure 9 . upper panel), well in agreement with the blackbody component seen in the spectra (Drake et al. 2011). By comparing with the average trend of temperature evolution of the hydrogen-poor superluminous supernovae (SLSNe I) and type II SNe (e.g., Inserra et al. 2018), we found that CSS1029 displays considerably higher temperature (by a factor of $\sim 1.5$ ) than the $\mathrm{SNe}$ at the same epoch ( 40-90 days since the peak). The dramatic temperature evolution for the $\mathrm{SNe}$ is likely resulting from cooling due to rapid expansion and radiative losses.

\subsubsection{MOSFIT fittings to the optical light curve of outburst}

We further explored whether the optical light curve of outburst can be fitted with the Monte Carlo software MOSFIT, which was recently applied to model the light curves of TDEs (Mockler et al. 2019). This has not been done in the previous work (D11). The TDE model in MOSFIT assumes that emission produced within an elliptical accretion disk of a TDE is partly reprocessed into the UV/optical by an optically thick layer (Guillochon et al. 2018). We run MOSFIT using a variant of the emcee ensemble-based Markov Chain Monte Carlo routine, until the fit has converged by reaching a potential scale reduction factor of $<1.2$ (Mockler et al. 2019). In Figure 10, we show the hostsubtracted V-band light curve, and an ensemble of model realizations from MOSFIT. The model is able to reproduce the data quite well, including the stages of the rise to peak, near the peak and steady decline at later times. To quantify how well the various combinations of parameters in the modelling, MOSFIT uses the Watanabe-Akaike information criteria (WAIC; Gelman et al. $20145^{5}$ For CSS1029, we found WAIC = 119, which is fairly consistent with the best-fit values for a sample of optical TDEs (Mockler et al.2019), suggesting that the fitting result is acceptable.

The best-fit parameters of the TDE model with the corresponding systematic and statistical errors at $1 \sigma$ confidence are shown in Table 4. Figure 11 shows the posterior probability distribution of the most relevant model parameters in the fit. The best-fit model is that of a black hole of $2.1 \times 10^{7} \mathrm{M}_{\odot}$ disrupting a star of $0.98 \mathrm{M}_{\odot}$. This black hole mass is consistent within errors with the mass estimated by Blanchard et al. (2017) using the $H_{\alpha}$ broad emission line. In comparison with other TDEs modeled in Mockler et al. (2019), the stellar mass for CSS1029 is larger than most other TDEs where a disrupted star with mass near $0.1 \mathrm{M}_{\odot}$ is preferred. As Table 4 shows, the best-fit model from MOSFIT indicates that the star was likely partially disrupted as the impact parameter $b<1$ (Mockler et al. 2019). This is similar to the case in the TDE AT2018hyz (Gomez et al. 2020), which has a $b=0.4$.

5 WAIC is a widely applicable Bayesian criteria, and evaluated as WAIC $=\left\langle\log p_{n}\right\rangle-\operatorname{var}\left(\log p_{n}\right)$, where $\left\langle\log p_{n}\right\rangle$ is the mean of $\log$ likelihood score and $\operatorname{var}\left(\log p_{n}\right)$ is its variance. 


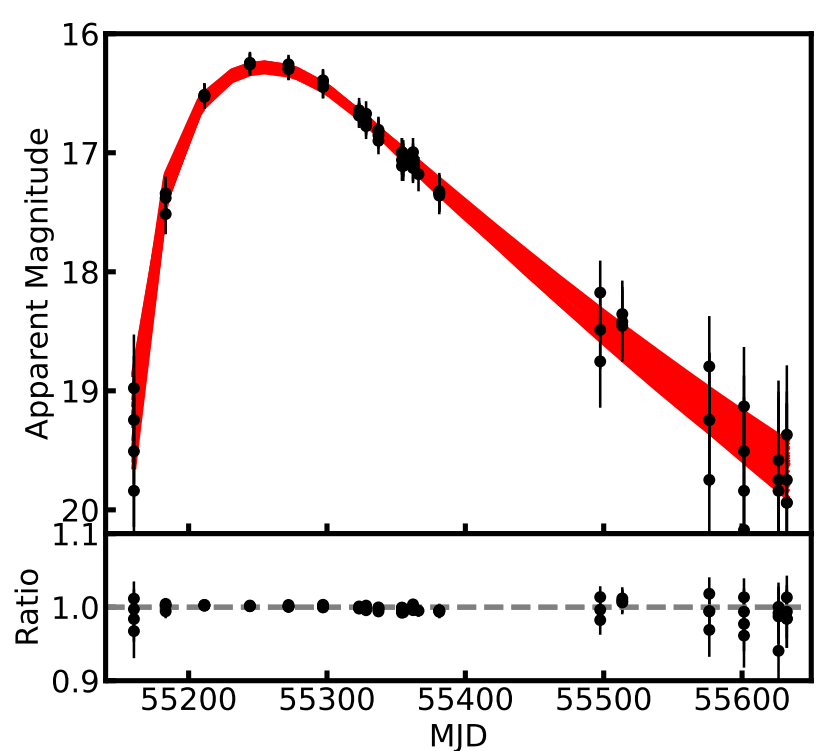

Fig. 10. Host-subtracted V-band light curve of CSS1029 (filled circles), and the best model realizations from MOSFIT (red curves). The most relevant best-fit model parameters are listed in Table 4.

Table 4. Best-fit model parameters from MOSFIT.

\begin{tabular}{cccc}
\hline \hline Parameter & Prior & Value & Units \\
\hline $\log \left(\mathrm{R}_{\mathrm{ph} 0}\right)$ & {$[-4,4]$} & $3.18_{-0.69}^{+0.53}$ & \\
$l$ & {$[0,4]$} & $1.52_{-0.39}^{+0.43}$ & \\
$\log \left(\mathrm{T}_{\text {viscous }}\right)$ & {$[-3,5]$} & $-0.67_{-1.1}^{+1.3}$ & days \\
$\mathrm{b}(\operatorname{scaled} \beta)$ & {$[0,2]$} & $0.61_{-0.03}^{+0.04}$ & \\
$\log \left(\mathrm{M}_{\mathrm{BH}}\right)$ & {$[5,8]$} & $7.33_{-0.06}^{+0.06}$ & $M_{\odot}$ \\
$\log \varepsilon$ & {$[-2,-0.4]$} & $-1.26_{-0.07}^{+0.07}$ & \\
starmass & {$[0.01,10]$} & $0.98_{-0.03}^{+0.02}$ & $M_{\odot}$ \\
$\log \sigma$ & {$[-4,2]$} & $-1.08_{-0.11}^{+0.11}$ & \\
\hline \hline
\end{tabular}

Notes. The parameter $R_{p h 0}$ and $l$ is the radius normalization and powerlaw exponent to compute the luminosity-dependent radius of the photosphere in MOSFIT; $T_{\text {viscous }}$ is the viscous time of the gas accretion onto the black hole; $b$ is a proxy for $\beta, \beta=R_{t} / R_{p}$, where $R_{t}$ refers to the tidal disruption radius and $R_{p}$ refers to the pericenter radius. Full stellar disruption corresponds to $b=1 ; M_{h}\left(M_{\odot}\right)$ is the black hole mass; $\varepsilon$ is the efficiency parameter that converts the input fallback rate of material to luminosity; starmass is the mass of disrupted star; $\sigma^{2}$ represents the additional variance in the Bayesian analysis to make $\chi_{r}^{2}=1$. The errors are only for the fitting statistical uncertainties, and do not include the systematic uncertainties from MOSFIT (Mockler et al. 2019).

\section{Discussion and Conclusion}

\subsection{Nature of optical outburst}

Since its discovery, the nature of optical outburst shown in the CRTS V-band lightcurve (Figure 8) is in dispute. D11 explored an origin of optical outburst as an AGN flare, a TDE, or a Type IIn SLSN, and argued for the last option that has to occur within $150 \mathrm{pc}$ of the nucleus of the galaxy. The normal AGN variability was ruled out as such a rapid (duration of $\sim 1 \mathrm{yr}$ ), energetic optical flare (total integrated energy of $\sim 10^{52} \mathrm{erg}$ ) is rarely seen in the light curves of NLS1 (D11; Kankare et al.2017).

On the other hand, the MIR outbursts in CSS1029 are clearly detected with WISE. In both the cases of SNe and TDE, strong

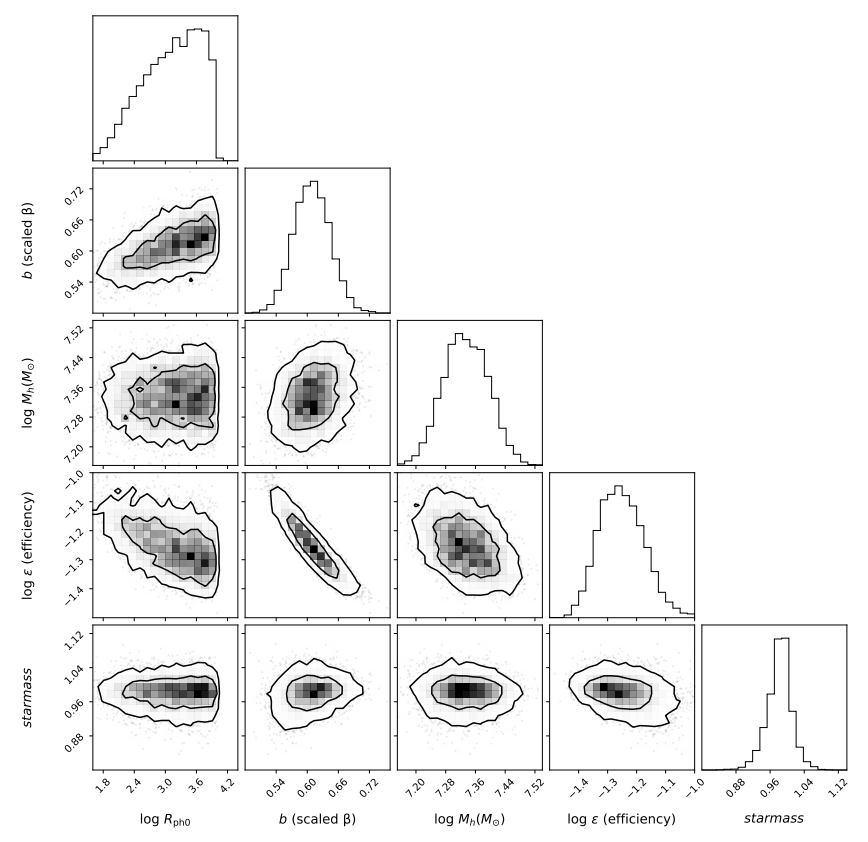

Fig. 11. Posterior distributions of model parameters from the fit to the V-band light curve of CSS1029, shown in Figure 10 The contours (from inside to out) indicate the $1 \sigma$ and $2 \sigma$ confidence intervals for two parameters of interest, respectively.

MIR flares are generally thought to be thermal emission from the dust heated by the nuclear optical outburst. The optical emission from the outburst can sublimate the dust within a certain range and form a dust cavity, the radius of which can be described by the sublimation radius $r_{\text {sub }}$. Assuming a dust sublimation temperature of $1800 \mathrm{~K}$ and a grain radius of $0.1 \mu \mathrm{m}$, the peak bolometric luminosity of the outburst of $4 \times 10^{44} \mathrm{erg} \mathrm{s}^{-1}$ in CSS1029 suggests a $r_{\text {sub }}$ of $\sim 0.3$ light-years The IR emitting dust has a distance $r \gtrsim r_{\text {sub }}$ from the central source, causing the IR emission delayed relative to optical emission. If the dust is heated directly by central UV/optical radiation, the time delay $\tau \sim r / c$ would be expected as several months. Conversely, if the dust is shock-heated by the material moving outward at a speed of $v_{\text {out }}$ (typically $\sim 0.02-0.1 \mathrm{c}$ ), from either the ejecta for $\mathrm{SNe}$ or outflow for TDE, the time delay $\tau \sim r / v_{\text {out }}$ would be several years or longer. Since the MIR outburst in CSS1029 was detected several months after the optical peak, the dust should be heated directly by radiation. The possibility of non-thermal radiation from a relativistic jet to cause the MIR flare can be ruled out, because the galaxy is radio quiet $\left(f_{6 \mathrm{~cm}} / f_{4400 \AA} \lesssim 1\right.$, D11). The host-subtracted peak MIR luminosity of CSS1029 is as high as of $1.26 \times 10^{44} \mathrm{erg}$ $\mathrm{s}^{-1}$. As shown in Figure 12, the peak MIR luminosity is among brightest ones for MIR nuclear transients, which is about two orders of magnitude higher than typical SNe explosions (Jiang et al. 2019, 2021, Szalai et al. 2019), and one order of magnitude higher than the rare population of SLSNe explosions, such as ASASSN-15lh (Dong et al. 2016). The peak MIR luminosity is close to those of several TDE candidates, including F010042237 (Dou et al. 2017), Arp 299B-AT1 (Mattila et al. 2018) and

${ }^{6}$ Here we used the formula given in Namekata \& Umemura (2016) to estimate the sublimation radius

$r_{\text {sub }}=0.121 p c\left(\frac{L_{\text {bol }}}{10^{45} \text { erg s}^{-1}}\right)^{0.5}\left(\frac{T_{\text {sub }}}{1800 K}\right)^{-2.804}\left(\frac{a}{0.1 \mu m}\right)^{-0.51}$. 
PS1-10adi (Jiang et al. 2019). Thus the high MIR luminosity prefers to the TDE interpretation of the outburst. Note that because of the sparse sampling of the MIR photometry during the outburst phase, the true peak luminosity for CSS1029 may be even higher. Furthermore, the optical peak luminosity matches the Eddington luminosity of the SMBH (Blanchard et al. 2017), supporting its association with accretion.

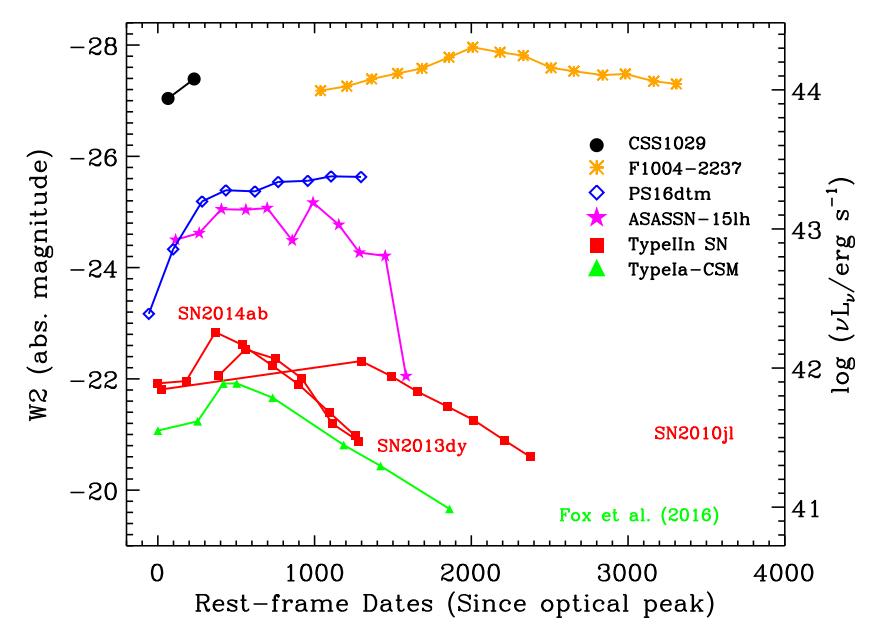

Fig. 12. Host-subtracted WISE W2 $(4.6 \mu \mathrm{m})$ magnitude for CSS1029 during outburst. For comparison, we also plot the evolution of peak MIR luminosity of the TDE F01004-2237 (Dou et al. 2017) and PS16dtm (Blanchard et al. 2017), SLSNe (Jiang et al.2019) and the supernovae with an excess of MIR emission, i.e., Type Iax SN 2014dt (Fox et al. 2016).

In Section 3.5.1, we have shown that CSS1029 exhibits a roughly constant temperature of $T \simeq 11300 \mathrm{~K}$ between $\sim$ 40 - 90 days since the optical peak. This is neither expected from the photosphere evolution of type II SNe, nor observed for hydrogen-poor SLSN (Inserra et al. 2018). For a typical SNe both its temperature and bolometric luminosity drop roughly as power-laws with time during the early stage ( $~ 1-3$ weeks), possibly as a result of cooling due to rapid expansion and radiative losses (e.g., Faran et al. 2018). Once the hydrogen recombination becomes important, which dominates the drop in the optical depth of the photosphere expansion, the temperature remains almost constant at $\sim 6000-7000 \mathrm{~K}$. With a temperature that is higher than averaged $\mathrm{SNe}$ by a factor of $\sim 1.5$ at the same epoch (Figure 9), this suggests that CSS1029 might be different from typical SNe explosions. However, the TDE candidate ASASSN-14ae showed a very similar temperature evolution to CSS1029. In fact, most TDEs discovered in the optical bands exhibit relatively flat temperature evolution over several months since peak (van Velzen et al.|2021; Holoien et al.|2021). The constant temperature evolution could be explained if the UV/optical emission is powered by the reprocessed accretion radiation by unbound TDE outflow (Metzger \& Stone 2016). This similarity between the evolution of CSS1029 and TDEs suggests that they might be due to the same mechanism.

On the other hand, the temperature for CSS1029 is on the low end of the temperature range observed for TDEs (of $\left.\sim 1-4 \times 10^{4} \mathrm{~K}\right)$. van Velzen et al. (2021) found a significant anti-correlation between the blackbody radius and temperature, following the relation $L_{\mathrm{bb}} \propto R^{2} T^{4}$. By fitting to the UV to optical SED, we found that the blackbody radius of CSS1029 is $\sim 6-8 \times 10^{15} \mathrm{~cm}$ (Figure B.2 in Appendix B), on the high end of the radius range observed for TDEs (Holoien et al. 2021). If assuming that the blackbody photospheric radius is proportional to the size of accretion disk in the context of TDEs, the large blackbody radius of CSS1029 could be attributed to the higher mass of disrupted star (van Velzen et al. 2021). This is consistent with the results of MOSFIT fittings to the V-band light curve (Table 4), where a black hole of $2 \times 10^{7} \mathrm{M}_{\odot}$ disrupting a star of $0.98 \mathrm{M}_{\odot}$ is inferred.

Note that D11 argued against a TDE explanation primarily because the blackbody temperature and flux decline rate were inconsistent with theoretical predictions from early studies of TDEs. However, as we shown in Section 3.1, the exponential decline rate that can best describe the outburst evolution of CSS1029 has also been observed in many TDEs that are discovered in modern optical surveys (e.g., Holoien et al. 2014, 2016, Shu et al. 2020). In addition, as shown in Section 3.4, our spectroscopy follow-up observations have revealed the brightening of the broad $\mathrm{H} \alpha$ flux, about a decade after the optical outburst. Although a broad $\mathrm{H} \alpha$ emission component has been observed to emerge in the late-time spectra of some SLSNes (Gal-Yam 2019), the time-scale for the appearance is much shorter, typically $\sim 1 \mathrm{yr}$. This makes the origin of late-time $\mathrm{H} \alpha$ brightening in CSS1029 distinct from what is expected in SLSNe, likely related to a TDE. Given the high peak MIR luminosity and relatively constant temperature evolution of $T \sim 11000 \mathrm{~K}$ over $\sim 40-90$ days since optical peak, we conclude that a TDE origin is preferred for the optical outburst for CSS1029 and the scenario with a superluminous $\mathrm{SNe}$ explosion seems disfavored.

\subsection{Possible origins for the $X$-ray brightening}

By analyzing the follow-up observations with Swift taken on 2015 and 2020, we found an X-ray brightening of $\gtrsim 30$ in CSS1029 relative to its previous low state in 2010, followed by a flux fading within about two weeks. The appearance of bright $\mathrm{X}$-ray emission at later times and its rapid decline in flux within a short period appear to be an unusual property of CSS1029. Since it was established previously that the optical spectrum of CSS1029 is that of NSy1 (D11), this may suggest that the Xray brightening could be attributed to the AGN activity. This is supported by the detection of a hard X-ray component with 2$10 \mathrm{keV}$ luminosity of $8.9_{-5.1}^{+6.7} \times 10^{42} \mathrm{erg} \mathrm{s}^{-1}$ (Section 3.2), and a best-fit photon index of $\Gamma \stackrel{-5.1}{\sim} 2.1$ typically for NSy1s. However, we note that the large-amplitude X-ray variability in CSS1029 is probably distinct from the rapid AGN variability seen on shorter time-scales of hours to days (e.g., Uttley et al. 1999:7]

As mentioned in Section 3.3, the UV emission shows little variability during the $\mathrm{X}$-ray flaring, with maximum variability amplitude of $\sim 50 \%$, which is mild compared with the X-ray one. Moreover, as shown in Figure 8, we do not find any coincident variability in either optical or MIR with the X-ray flaring in 2015. Note that similar X-ray flares have been observed in the AGN IC 3599 which could be attributed to an instability in a radiation-pressure dominated accretion disk (Grupe et al. 2015), or multiple tidal disruption flares with the recurrence time of 9.5 yr (Campana et al. 2015). However, in this case a large optical outburst was also observed prior to the X-ray one, making it physically different from what is observed in CSS1029. This

\footnotetext{
7 From the extinction-corrected [O $\mathrm{III}] \lambda 5007$ luminosity of $2.4 \times$ $10^{41} \mathrm{erg} \mathrm{s}^{-1}$ from the SDSS spectrum (D11), we roughly estimated the "time-averaged" $2-10 \mathrm{keV}$ luminosity of $3.0_{-0.6}^{+0.8} \times 10^{42} \mathrm{erg} \mathrm{s}^{-1}$ (e.g., Lamastra et al. 2009). This is a factor of $\sim 3$ higher than the upper limit of hard X-ray luminosity obtained in 2010, suggesting that CSS1029 could be in a historically low X-ray state during the optical outburst.
} 
suggests that the scenario that the dramatic change in AGN accretion rate causing the extreme X-ray variability in CSS1029 seems unlikely. The microlensing by stars in foreground galaxies seem also unable to explain the observed X-ray behavior, as the model predicts a variability that is independent of wavelengths (e.g., Lawrence et al. 2016).

The apparent lack of coincident optical/UV variability with $\mathrm{X}$-ray flaring in CSS1029 is somewhat similar to that found in some NSy1s with extreme X-ray variations (e.g., Bachev et al. 2009; Miniutti et al. 2012; Grupe et al.|2008, Buisson et al. 2018; Grupe et al. 2019). It is suggested that the absorber with a nonunity covering fraction at large scale can cause the X-ray variability, but it requires to be dust-free so as not affecting the UV or optical emission. Assuming a single large cloud passing over the X-ray source, we can use the observed duration of X-ray flux declining in 2015 (at least $10 \mathrm{~d}$, Table 1) to constrain the location of the absorber. The crossing time for an intervening cloud on a Keplerian orbit can be estimated as

$t_{\text {cross }}=\left(G M_{\mathrm{BH}}\right)^{-1 / 2} r_{c}^{1 / 2} D_{\mathrm{src}}$

where $r_{c}$ is the distance of the cloud to $\mathrm{X}$-ray source, $D_{\text {src }}$ is size for the X-ray source and $t_{\text {cross }} \approx D_{\text {src }} / V_{K}\left(V_{K}\right.$ is Keplerian velocity, Risaliti et al. 2007). For a BH mass of $7.9 \times 10^{6} \mathrm{M}_{\odot}$ (Blanchard et al. 2017) and a typical diameter of $10 R_{\mathrm{G}}$ for the X-ray source (e.g., Fabian et al. 2009), we find the location of intervening cloud $r_{c} \geq 5 \times 10^{18} \mathrm{~cm}$, in order to have an obscuration that lasts longer than $10 \mathrm{~d}$. This corresponds to $\sim 1.6 \mathrm{pc}$, which is suggestive of a cloud that lies outside of the BLR. Note that this distance is much larger than the typical dust sublimation radius in AGNs ( 0.1pc, Jiang et al. 2019), making the explanation for the presence of dust-free clouds at such a large distance challenging. Therefore, the scenario that X-ray brightening in CSS1029 due to the movement of dust-free absorbing gas at large scale seems disfavored.

Having established that TDE is plausible to power the optical outburst, we discuss the possibility of whether a TDE could contribute to the X-ray variability in CSS1029. Hydrodynamics simulations have shown that the debris stream of a TDE can affect the accretion properties of pre-existing AGN (Chan et al. 2019). Blanchard et al. (2017) presented the observation of PS16dtm, a TDE occurred at the nucleus of a NSy1, which shares many similarities with CSS1029. Blanchard et al. (2017) suggested that the TDE can lead to the dimming of the X-ray emission from the pre-existing AGN, possibly due to the obscuration of X-ray emitting region by stellar debris. They further predicted that the source will become X-ray bright again approximately a decade since the TDE, though it has not been confirmed yet. In comparison with PS16dtm, the relatively faint X-ray flux of CSS1029 observed in 2010 could simply due to the partial obscuration of X-ray emitting region by accreting stellar debris, and the brightening in 2015 could be attributed to the decrease in the optical depth of absorbing gas as the accretion rate declines.

On the other hand, Ricci et al. (2020) found the clear evidence that the hard power-law component (produced in X-ray corona) disappeared after the UV/optical outbursts in the AGN $1 \mathrm{ES} 1927+65$, i.e., drops in luminosity by more than three orders of magnitude. Interestingly, such a power-law component reappeared about one year later. Ricci et al. (2020) invoked the TDE scenario to explain the drastic X-ray variability, which can deplete the innermost regions of accretion flow (Chan et al. 2019), hence disrupting the magnetic field powering the X-ray corona. If the relative X-ray faintness of CSS1029 in 2010 is due to the destruction of AGN X-ray corona, its X-ray luminosity
$\left(L_{0.3-2 \mathrm{keV}} \sim 3.7 \times 10^{42} \mathrm{erg} \mathrm{s}^{-1}\right)$ appears too high in comparison to what is observed in 1ES1927+65. In this case, the observed X-ray emission of CSS1029 in 2010 could be attributed solely to the TDE.

Alternatively, several works have invoked a partial covering model to account for extreme X-ray variability behaviors of AGNs, especially those high accreting systems (Luo et al. 2015. Liu et al. 2019; Ni et al. 2020). In this model, the central X-ray source could be partially obscured by a thick inner disk or outflow if it intercepts the line of sight. Similar scenario has been proposed to explain the faintness of the X-ray emission in optical TDEs by the orientation effect where the X-rays from the inner disk is obscured by dense outflow gas (Dai et al. 2018). The slight changes in the covering factor with respect to the $\mathrm{X}$-ray source would result in an X-ray variability, but the bulk UV/optical continuum at outer disk remains little affected. Indeed, Blanchard et al. (2017) estimated that the Eddington ratio is close to 1 for CSS1029 when it reaches to the peak luminosity of outburst on 2010, indicating the central $\mathrm{BH}$ is accreting at a high rate during this epoch. Simulations have suggested that geometrically thick inner accretion disks and associated dense outflows will be formed when accretion rate is high enough (Jiang et al. 2014, 2019). This is suggestive of the possibility that a thick disk in the inner region formed in CSS1029 plays a role in partially obscuring central X-ray source, explaining the low X-ray flux observed in 2010. The X-ray brightening observed at later times may be due to a decrease in the thickness or covering factor of the inner disk with accretion rate (radiation luminosity), possibly induced by the TDE process. As shown in Figure 8 . the baseline AGN optical emission decreased by a factor of $\sim 0.3$ mag after the outburst seems to be in accordance with this scenario.

\section{3. $H \alpha$ anomaly and implications for the BLR evolution}

In Section 3.4, our analysis suggests transient $\mathrm{H} \alpha$ brightening in CSS1029 that might not be relevant to the continuum variation. Although we cannot locate spectra taken between 2018 and 2020, the most recent photometric observations by Swift show that the amplitude of continuum variability at UV/optical bands was not as large as that expected from the $\mathrm{H} \alpha$ brightening (Table 1). The lack of corresponding ionizing continuum variability is further supported by the WISE light curves at MIR bands (Figure 8), which suggest no recent outburst even for the largely unobservable extreme-UV variation up to Dec 2020. Otherwise an MIR echo signal might be detected. We argue for that the "non-responsivity" of ionizing continuum variability is not due to the contamination of constant host emission, as the optical continuum is dominated by the AGN emission in the spectroscopic data (Section 3.4). The clear flux decline in the V-band light curve by $\sim 0.3 \mathrm{mag}$ (Figure 8 ) is also suggestive of the dominant AGN contribution to the optical photometry. Therefore, the observed $\mathrm{H} \alpha$ brightening is an anomalous behavior of the BEL variations in the context of RM model. Note that transient anomalous phenomenon has been reported in other AGNs from the RM campaign (Goad et al. 2016, Gaskell et al. 2021), but most are characterized by a significant deficit in the flux of BELs when continuum is brightened, which is clearly different from the $\mathrm{H} \alpha$ anomaly observed in CSS1029.

As no clear evidence for a temporary increase in the ionizing continuum incident upon BLR clouds is found during/before the anomaly, it could be possible to explain the enhanced $\mathrm{H} \alpha$ emission by an increase of gas density or excitation within BLR itself. Although the formation processes of the BLR are still unknown, 
Czerny \& Hryniewicz (2011) suggested that the BLR is a failed dusty wind from outer accretion disk. Baskin \& Laor (2018) refined this model by exploring the dust properties as well as implied BLR structure in more detail, and proposed a dust inflated accretion disk as the origin of the BLR. The dusty disk wind scenario may not be able to explain the $\mathrm{H} \alpha$ anomaly, as it requires a replenishment of dusty clouds emerged from accretion disk, resulting in a brightening in the IR emission before the $\mathrm{H} \alpha$ enhancement which is not yet observed. On the other hand, Wang et al. (2017) suggested that tidally disrupted clumps within the dust sublimation radius of the torus by central black hole may become bound at smaller radii to serve the source of the BLR gas. In such a model, the $\mathrm{H} \alpha$ anomaly in CSS1029 could be induced by a sudden increase in the tidal disruption rate, or in the total mass of tidally disrupted clumps, supplying as addition to the BLR gas clouds. However, the mechanism by which the tidal disruption rate is increased abruptly is not clear in this scenario. In addition, one would ask why the similar behavior of the BEL variations has not been observed in other AGNs for which intense RM monitoring campaigns are available.

As we have demonstrated, CSS1029 represents one of few AGNs in which a TDE-like energetic transient event was discovered (e.g., Kankare et al. 2017; Blanchard et al. 2017, Shu et al. 2018; Liu et al. 2020). While the stellar disruption itself may be less affected by the AGN accretion disk, the bound debris stream could collide with the disk, exciting shocks and leading to inflow and considerable energy dissipation in the disk as well as the coronal region responsible for the most AGN X-ray emission (Blanchard et al. 2017, Chan et al. 2019, Ricci et al. 2020). On the other hand, it has been proposed that when star is disrupted, roughly half the stellar debris is unbound, and ejected with an estimated velocity $v_{\mathrm{ej}} \sim\left(2 G M_{\mathrm{BH}} R_{\star} / R_{\mathrm{p}}^{2}\right)^{1 / 2}($ Evans \& Kochanek 1989). For a solar star and with $M_{\mathrm{BH}}=7.9 \times 10^{6} \mathrm{M}_{\odot}(\mathrm{Sec}-$ tion 4.3) for CSS1029, the maximum ejection velocity is of $v_{\mathrm{ej}} \sim 8 \times 10^{3} \mathrm{~km} \mathrm{~s}^{-1}$. The ejection of unbound debris may run into the BLR, providing an alternative supply for the BLR gas to explain the $\mathrm{H} \alpha$ anomaly. In fact, the geometry and energetics of the ejected material and possible observational consequences of the interaction of this material with the ambient medium surrounding the black hole have been investigated theoretically (Rees 1988, Kochanek 1994, Khokhlov \& Melia 1996, Guillochon et al.2016). Given an expected BLR size of $r_{\mathrm{BLR}} \sim 30$ light days from the 5100 $\AA$ luminosity, the timescale for debris to spread out and reach the BLR is $r_{\mathrm{BLR}} / v_{\mathrm{ej}}=3.1$ years. However, TDE simulations have shown that the average kinetic energy for ejecta is about an order of magnitude lower than expected, resulting in a typical outgoing velocity of $\sim 2500 \mathrm{~km} \mathrm{~s}^{-1}$ (Guillochon \& Ramirez-Ruiz 2013). This corresponds to a timescale of $\sim 10$ years for debris moving to BLR, which is not inconsistent with the time when the $\mathrm{H} \alpha$ enhancement was observed in CSS1029. It is intriguing to note that the changes in the flux of higher-order $\mathrm{H} \beta$ line are less than the $\mathrm{H} \alpha$ (Figure 6), indicating that the gas replenishment alone may not be able to account for the spectral evolution of CSS1029, as a larger responsivity of $\mathrm{H} \beta$ is expected with decreasing of ionization parameter (Korista \& Goad 2004). This suggests that in addition to photoionization, collisional excitation might be important for producing $\mathrm{H} \alpha$. Better quantitative modeling of the interaction of stellar debris with BLR might be required to explain the anomalous enhancement of $\mathrm{H} \alpha$ emission, which is beyond the scope of this paper.

Since the anomalous behavior of this kind of BEL variation is rare in AGNs, spectroscopic monitoring campaigns of CSS1029 with contemporaneous multi-wavelength photometric observations are encouraged. In addition, observations of similar BEL anomaly in other AGNs from dedicated RM campaigns are important to constrain whether this is unique for CSS1029 (e.g., due to the TDE influence) or common phenomenon among AGNs. Such datasets could potentially reveal the underlying physical processes that drive the (de)correlation between continuum and BEL variations, yielding new insights into the formation and evolution of BLR.

Acknowledgements. We thank the Swift Acting PI, Brad Cenko, for approving our ToO request to observe CSS1029, Matthew J. Graham, Andrew J. Drake for kindly providing the post-DR2 CRTS data for the source, and Pu Du for timely coordinating the Lijiang/YFOSC observations. This research made use of the HEASARC online data archive services, and data products from the Wide-field Infrared Survey Explorer, Catalina Real-time Transient Survey and Zwicky Transient Facility Project. We acknowledge the use of the Hale 200-inch Telescope through the Telescope Access Program (TAP), under the agreement between the National Astronomical Observatories, CAS, and the California Institute of Technology, and the support of the staff of the Lijiang $2.4 \mathrm{~m}$ telescope. Funding for the Lijiang telescope has been provided by CAS and the People's Government of Yunnan Province. This work is supported by Chinese NSF through grant Nos. 11822301, 12192220, 12192221, 11833007, and U1731104.

\section{References}

Auchettl, K., Guillochon, J., \& Ramirez-Ruiz, E. 2017, ApJ, 838, 149 Auchettl, K., Ramirez-Ruiz, E., \& Guillochon, J. 2018, ApJ, 852, 37 Bachev, R., Grupe, D., Boeva, S., et al. 2009, MNRAS, 399, 750 Baskin, A., \& Laor, A. 2018, MNRAS, 474, 1970 Blandford, R. D., \& McKee, C. F. 1982, ApJ, 255, 419

Blanchard, P., Nicholl, M., Berger, E., \& Guillochon, J. 2017, ApJ, 843, 106B Boller, T., Schmitt, J. H. M. M., Buchner, J., et al. 2021, arXiv:2106.14523 Boroson, T. A., \& Green, R. F. 1992, ApJS, 80, 109

Buisson, D. J. K., Lohfink, A. M., Alston, W. N., et al. 2018, MNRAS, 475, 2306 Chan, C.-H., Piran, T., Krolik, J. H., et al. 2019, ApJ, 881, 113 Campana, S., Mainetti, D., Colpi, M., et al. 2015, A\&A, 581, A17 Czerny, B., \& Hryniewicz, K. 2011, A\&A, 525, L8

Dai, L., McKinney, J. C., Roth, N., et al. 2018, ApJ, 859, L20 Dong, S., Shappee, B. J., Prieto, J. L., et al. 2016, Science, 351, 257 Dou, L., Wang, T., Yan, L., et al. 2017, ApJ, 841, L8

Drake, A. J., Djorgovski, S. G., Mahabal, A., et al. 2009, ApJ, 696, 870 Drake, A. J., Djorgovski, S. G., Mahabal, A., et al. 2011, ApJ, 735, 106 Elvis, M., Wilkes, B. J., McDowell, J. C., et al. 1994, ApJS, 95, 1 Evans, C. R., \& Kochanek, C. S. 1989, ApJ, 346, L13 Fabian, A. C., Zoghbi, A., Ross, R. R., et al. 2009, Nature, 459, 540 Fabian, A. C., Zoghbi, A., Wilkins, D., et al. 2012, MNRAS, 419, 116 F Fabian, A., Parker, M., Wilkins, D., \& Miller, J. 2014, MNRAS, 439, 2307F Faran, T., Nakar, E., \& Poznanski, D. 2018, MNRAS, 473, 513 Fox, O. D., Johansson, J., Kasliwal, M., et al. 2016, ApJ, 816, L13 Gallo, L. C., Grupe, D., Schartel, N., et al. 2011, MNRAS, 412, 161 Gelman, A., Hwang, J., \& Vehtari, A. 2014, Statistics and Computing, 24, 997 Gal-Yam, A. 2019, ARA\&A, 57, 305

Gibson, R. R., Brandt, W. N., \& Schneider, D. P. 2008, ApJ, 685, 773 Gaskell, C. M., Bartel, K., Deffner, J. N., et al. 2021, MNRAS, 508, 6077 Gierliński, M., Middleton, M., Ward, M., et al. 2008, Nature, 455, 369 Gilli, R., Maiolino, R., Marconi, A., et al. 2000, A\&A, 355, 485 Goad, M. R., Korista, K. T., De Rosa, G., et al. 2016, ApJ, 824, 11 Gomez, S., Nicholl, M., Short, P., et al. 2020, MNRAS, 497, 1925 González-Martín, O., \& Vaughan, S. 2012, A\&A, 544, A80 Graham, M. J., Djorgovski, S. G., Drake, A. J., et al. 2017, MNRAS, 470, 4112 Greene, J. E., \& Ho, L. C. 2005, ApJ, 630, 122

Grupe, D., Komossa, S., Gallo, L. C., et al. 2008, ApJ, 681, 982 Grupe, D., Komossa, S., Leighly, K. M., et al. 2010, ApJS, 187, 64 Grupe, D., Komossa, S., Leighly, K. M., et al. 2012, European Physical Journal Web of Conferences, 39, 06001. doi:10.1051/epjconf/20123906001 Grupe, D., Komossa, S., Gallo, L. C., et al. 2012, ApJS, 199, 28 Grupe, D., Komossa, S., \& Saxton, R. 2015, ApJ, 803, L28 Grupe, D., S.Komossa, L.Gallo, \& N.Schartel. 2019, MNRAS, 486, $227 \mathrm{G}$ Guainazzi, M., Nicastro, F., Fiore, F., et al. 1998, MNRAS, 301, L1 Guillochon, J. \& Ramirez-Ruiz, E. 2013, ApJ, 767, 25 Guillochon, J., Manukian, H., \& Ramirez-Ruiz, E. 2014, ApJ, 783, 23 Guillochon, J., McCourt, M., Chen, X., et al. 2016, ApJ, 822, 48 Guillochon, J., Nicholl, M., Villar, V. A., et al. 2018, ApJS, 23 Hinkle, J. T., Holoien, T. W.-S., Shappee, B. J., et al. 2021, arXiv:2108.03245 Holoien, T. W.-S., Prieto, J. L., Bersier, D., et al. 2014, MNRAS, 445, 3263 Holoien, T. W.-S., Kochanek, C. S., Prieto, J. L., et al. 2016, MNRAS, 463, 3813 
Holoien, T. W.-S., Neustadt, J. M. M., Vallely, P. J., et al. 2021, arXiv:2109.07480

Inserra, C., Smartt, S. J., Gall, E. E. E., et al. 2018, MNRAS, 475, 1046

Jiang, Y.-F., Stone, J. M., \& Davis, S. W. 2014, ApJ, 796, 106

Jiang, N., Dou, L., Wang, T., et al. 2016, ApJ, 828, L14

Jiang, Y., Davis, S., \& Stone, J. 2016, ApJ, 827, 10J

Jiang, N., Wang, T., Mou, G., et al. 2019, ApJ, 871, 15

Jiang, N., Wang, T., Dou, L., et al. 2021, ApJS, 252, 32

Jiang, Y.-F., Stone, J. M., \& Davis, S. W. 2019, ApJ, 880, 67

Kankare, E., Kotak, R., Mattila, S., et al. 2017, Nature Astronomy, 1, 865

Karas, V., \& Šubr, L. 2007, A\&A, 470, 11

Khokhlov, A. \& Melia, F. 1996, ApJ, 457, L61

Kochanek, C. S. 1994, ApJ, 422, 508

Komossa, S. 2015, Journal of High Energy Astrophysics, 7, 148

Korista, K. T., \& Goad, M. R. 2004, ApJ, 606, 749

Lamastra, A., Bianchi, S., Matt, G., et al. 2009, A\&A, 504, 73

Lawrence, A. 2018, NatAs, 2, 102L

Lawrence, A., Bruce, A. G., MacLeod, C., et al. 2016, MNRAS, 463, 296

Leloudas, G., Fraser, M., Stone, N. C., et al. 2016, Nature Astronomy, 1, 0002

Lin, D., Guillochon, J., Komossa, S., et al. 2017, Nature Astronomy, 1, 0033

Liu, H., Luo, B., \& Brandt, W. N. 2019, ApJ, 878, 79

Liu, Z., Li, D., Liu, H.-Y., et al. 2020, ApJ, 894, 93

Luo, B., W.N.Brandt, P.B.Hall, Wu, J., \& S.F.Anderson. 2015, ApJ, 805, 122

Mattila, S., Pérez-Torres, M., Efstathiou, A., et al. 2018, Science, 361, 482

Metzger, B. D., \& Stone, N. C. 2016, MNRAS, 461, 948

Miniutti, G., Fabian, A., Goyder, R., \& Lasenby, A. 2003, MNRAS, 344L, 22M

Middei, R., Vagnetti, F., Bianchi, S., et al. 2017, A\&A, 599, A82

Miniutti, G., \& Fabian, A. 2004, MNRAS, 349, 1435M

Miniutti, G., Brandt, W. N., Schneider, D. P., et al. 2012, MNRAS, 425, 1718

Miniutti, G., Sanfrutos, M., Beuchert, T., et al. 2014, MNRAS, 437, 1776

Miniutti, G., Saxton, R. D., Giustini, M., et al. 2019, Nature, 573, 381

Mockler, B., Guillochon, J., \& Ramirez-Ruiz, E. 2019, ApJ, 872, 151

Namekata, D. \& Umemura, M. 2016, MNRAS, 460, 980

Ni, Q., Brandt, W., Luo, B., Hall, P., \& Shen, Y. 2018, MNRAS, 480, 5184N

Ni, Q., Brandt, W. N., Yi, W., et al. 2020, ApJ, 889, L37

Oknyansky, V. L., Winkler, H., Tsygankov, S. S., et al. 2019, MNRAS, 483, 558

Parker, M. L., Komossa, S., Kollatschny, W., et al. 2016, MNRAS, 461, 1927

Peterson, B. M. 1993, PASP, 105, 247

Peterson, B. M., Denney, K. D., De Rosa, G., et al. 2013, ApJ, 779, 109

Rees, M. J. 1988, Nature, 333, 523

Ricci C., Kara E., Loewenstein M., Trakhtenbrot B., Arcavi I., Remillard R., Fabian A. C., et al., 2020, ApJL, 898, L

Ricci, C., Loewenstein, M., Kara, E., et al. 2021, ApJS, 255, 7

Risaliti, G., Elvis, M., Fabbiano, G., Baldi, A., \& Zezas, A. 2005, ApJ, 623, L93

Risaliti, G., Elvis, M., Fabbiano, G., et al. 2007, ApJ, 659, L111

Saxton, R., Read, A., Esquej, P., et al. 2011, Narrow-line Seyfert 1 Galaxies and Their Place in the Universe, 8

Sun, L., Shu, X., \& Wang, T. 2013, ApJ, 768, 167

Saxton, R. D., Motta, S. E., Komossa, S., et al. 2015, MNRAS, 454, 2798

Saxton, R., Motch, C., Komossa, S., et al. 2019, Astronomische Nachrichten, 340,351

Schlegel, D. J., Finkbeiner, D. P., \& Davis, M. 1998, ApJ, 500, 525

Shappee, B. J., Prieto, J. L., Grupe, D., et al. 2014, ApJ, 788, 48

Shapovalova, A. I., Popović, L. Ć., Burenkov, A. N., et al. 2012, ApJS, 202, 10

Shapovalova, A. I., Popović, L. Č., et al. 2019, MNRAS, 485, 4790

Shu, X., Wang, S., Dou, L., et al. 2018, ApJ, 857, 16S

Shu, X., Zhang, W., Li, S., et al. 2020, Nature Communications, 11, 5876.

Song, J. R., Shu, X. W., Sun, L. M., et al. 2020, A\&A, 644, L9

Steffen, A. T., Strateva, I., Brandt, W. N., et al. 2006, AJ, 131, 2826

Szalai, T., Zsíros, S., Fox, O. D., et al. 2019, ApJS, 241, 38

Trakhtenbrot, B., Arcavi, I., Ricci, C., et al. 2019, Nature Astronomy, 3, 242

Ulrich, M.-H., Maraschi, L., \& Urry, C. M. 1997, ARA\&A, 35, 445

Uttley, P., McHardy, I. M., Papadakis, I. E., et al. 1999, MNRAS, 307, L6

van Velzen, S., Anderson, G. E., Stone, N. C., et al. 2016, Science, 351, 62

van Velzen, S., Gezari, S., Hammerstein, E., et al. 2021, ApJ, 908, 4

Vestergaard, M., \& Wilkes, B. J. 2001, ApJS, 134, 1

Wang, J.-M., Du, P., Brotherton, M. S., et al. 2017, Nature Astronomy, 1, 775

Wevers, T. 2020, MNRAS, 497, L1

Wilkins, D. R., Gallo, L. C., Grupe, D., et al. 2015, MNRAS, 454, 4440

Yang, G., Brandt, W. N., Luo, B., et al. 2016, ApJ, 831, 145

Yip, C. W., Connolly, A. J., Vanden Berk, D. E., et al. 2004, AJ, 128, 2603 


\section{Appendix A: Test on the effects of seeing and slit width on the $\mathrm{H} \alpha$ variability}

In this section, we investigate whether the variations in the flux and $\mathrm{EW}$ of $H_{\alpha}$ broad emission line (BEL) from five long-slit spectroscopic observations can be caused by different seeing conditions or slit widths used to extract the spectrum. Such an effect could lead to changes in the fraction of emission that falls into the aperture. Firstly, we checked whether the spatial brightness profile of the $\mathrm{H} \alpha$ BEL and the continuum are different. In the case of significant difference in the spatial brightness profile between the two, the measured $\mathrm{H} \alpha \mathrm{EW}$ may deviate from the actual value. Since the BLR for an AGN at $z=0.147$ (the redshift of CSS1029) is expected to be a point-like source for groundbased observations, we can measure the spatial brightness profile of $\mathrm{H} \alpha \mathrm{BEL}$ along the slit, and then compare it to that of the continuum which presumably consists of both AGN and host component. To do so, we utilized the data from P200/DBSP observation taken on Feb 2020, as it has the best seeing of 1.5". The spatial brightness profile of continuum was extracted in two spectral windows around $\mathrm{H} \alpha$ with wavelength ranges of $6000-6500 \AA$ and $6640-6900 \AA$, while the profile of $\mathrm{H} \alpha$ BEL was extracted from the continuum-subtracted spectrum in the wavelength range of 6505-6615 $\AA$. Note that for extraction of $\mathrm{H} \alpha$ BEL, we masked two windows affected by narrow emission lines covering narrow wavelength ranges of $6559-6568 \AA$ and $6582-6588 \AA$. The results are shown in Figure A.1. It can be seen that the spatial brightness distribution of continuum is slightly extended compared to that of $\mathrm{H} \alpha \mathrm{BEL}$, the latter can be considered as a point-like source.

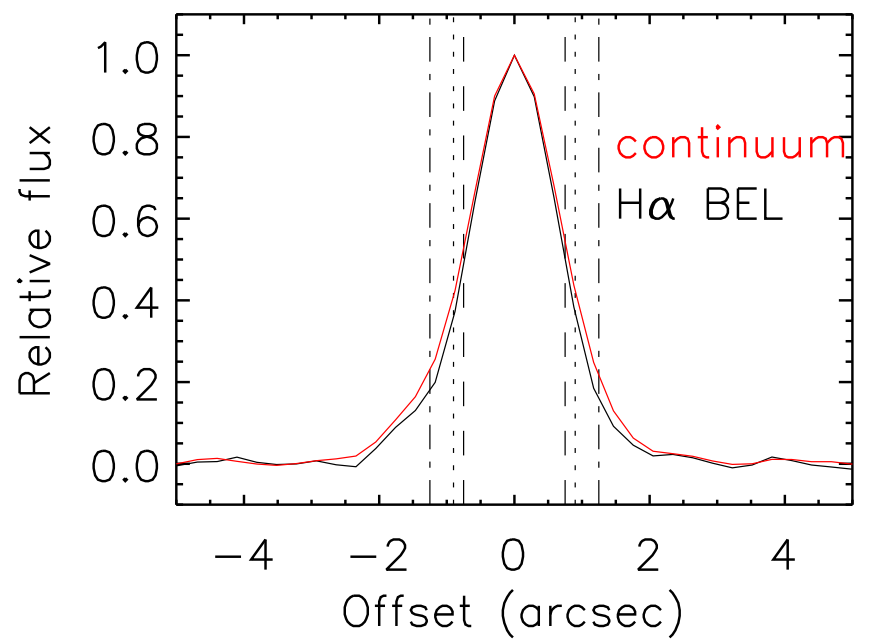

Fig. A.1. The brightness profile of $\mathrm{H} \alpha \mathrm{BEL}$ and underlying continuum along the slit, extracted from the data with the best seeing condition (Feb 2020). We also show the slit width of 1.5", 1.8" and 2.5" with dashed, dotted and dash-dotted line, respectively.

We demonstrate that the slight difference in the spatial distribution of continuum and $\mathrm{H} \alpha$ BEL does not cause a significant deviation of the measured $\mathrm{EW}$ of $\mathrm{H} \alpha$ using the following simulations. In the simulations, we assume that the $\mathrm{EW}$ of $\mathrm{H} \alpha$ remains constant (as the value observed in Feb 2020), and generate fake data that would be observed under different seeing conditions. We consider the effect of a worse seeing condition by convolving the brightness profile of $\mathrm{H} \alpha$ BEL with Gaussian functions. We then measure the fractions of $\mathrm{H} \alpha \mathrm{BEL}$ and continuum that fall into apertures. In Figure A.2, we show the ratio between the two under different seeing conditions for a slit width of $1.5^{\prime \prime}, 1.8^{\prime \prime}$ and $2.5^{\prime \prime}$, respectively. The ratios are nor- malized to the value measured in Feb 2020. This normalized ratio represents the deviation of the measured $\mathrm{EW}$ of $\mathrm{H} \alpha$ caused by changes in different seeing conditions and slit widths. The simulations show that the deviation is at most at a level of $7 \%$ for the seeing between $1.5^{\prime \prime}$ and $3.3^{\prime \prime}$, and the slit width between $1.5^{\prime \prime}$ and $2.5^{\prime \prime}$. Note that for the other four spectroscopic observations with worse seeing conditions, the deviation is expected smaller. Since the observed variations in the EW of $\mathrm{H} \alpha$ have a much larger amplitude than this value (up to $33 \%$ ), we conclude that the $\mathrm{H} \alpha$ variability is intrinsic and cannot be explained by the variations in either seeing or slit width.

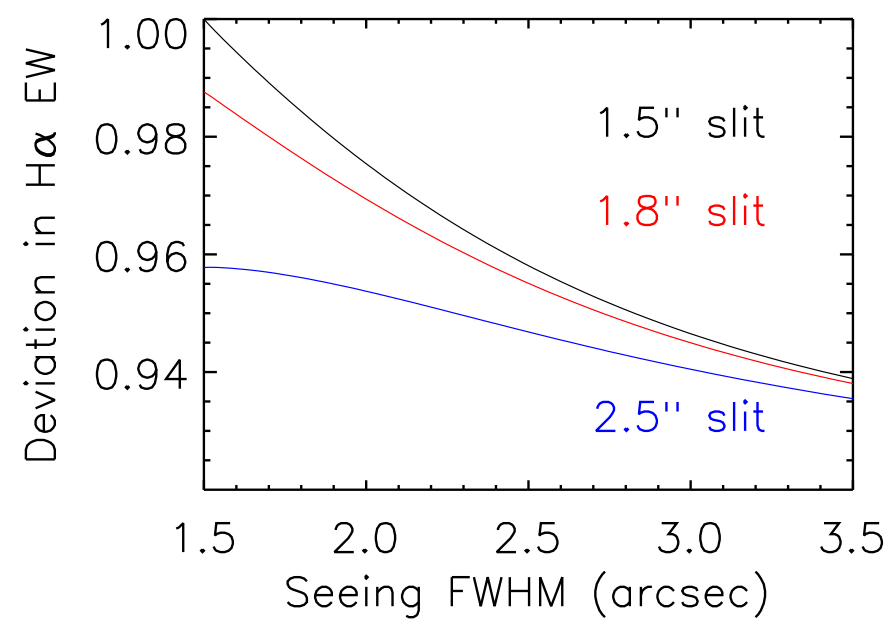

Fig. A.2. The deviation of measured $\mathrm{H} \alpha$ EW caused by the change in the seeing conditions using simulations, for the slit width of $1.5^{\prime \prime}$ (black), 1.8" (red) and 2.5" (blue), respectively.

\section{Appendix B: UV to optical SED fittings}

We fitted a blackbody model $\left(B_{v}=\frac{2 h v^{3}}{c^{2}} \frac{1}{e^{h v / k T}-1}\right)$ to the host subtracted, extinction-corrected photometric data from the Swift UVOT observations during the period of optical outburst, to put constrains on the luminosity, temperature and radius evolution of UV and optical emission. Uncertainties on the above parameters were derived using Monte Carlo simulations, in which the observed fluxes were randomly perturbed with amplitude by assuming Gaussian noise according to the photometric errors. This procedure was repeated 1000 times. The error bars on each parameter were then derived from the 16th and 84th percentiles of the distribution of the corresponding values obtained in the simulations. The UV to optical SED for different epochs, with its best-fitting blackbody model, are shown in Figure B.1. Using the best-fit temperature and rest-frame monochromatic UV luminosity at each epoch, we estimated the blackbody radius from $L_{v}=\pi B_{v} \times 4 \pi R_{\mathrm{BB}}^{2}$ and took blackbody luminosity from $L_{\mathrm{BB}}=\sigma T^{4} \times 4 \pi R_{\mathrm{BB}}^{2}$ as the integrated luminosity of UV and optical emission. The evolution of blackbody luminosity, temperature and radius are presented in the Figure B.2 

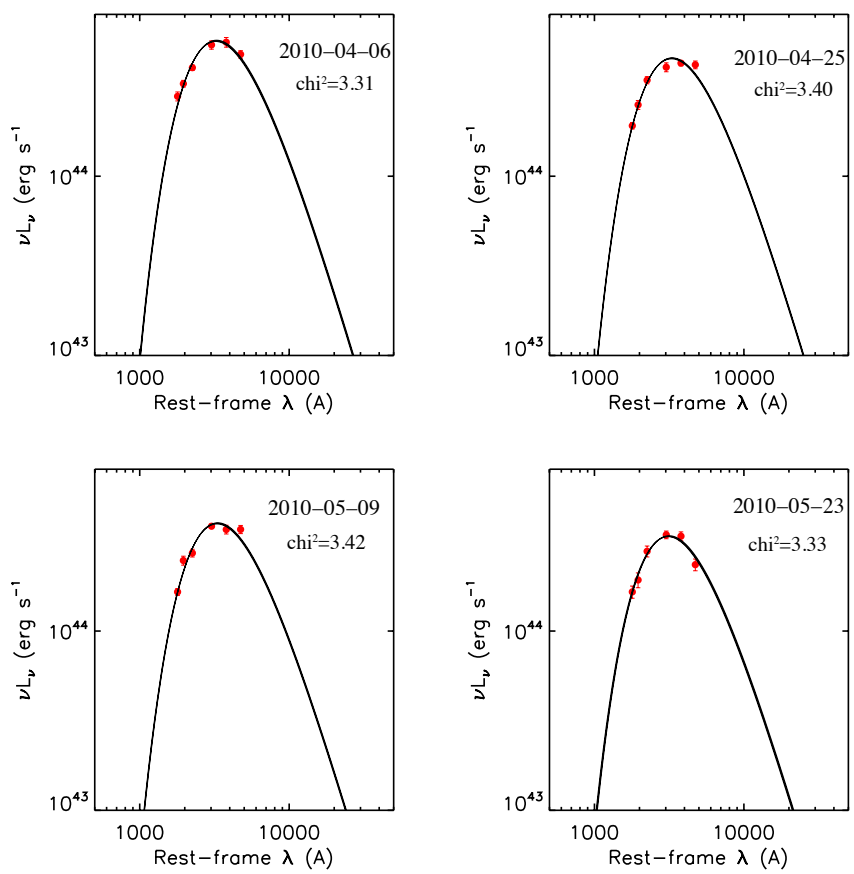

Fig. B.1. UV to optical SED obtained from the Swift UVOT observations during the period of the outburst, with the best-fit blackbody model for each epoch.

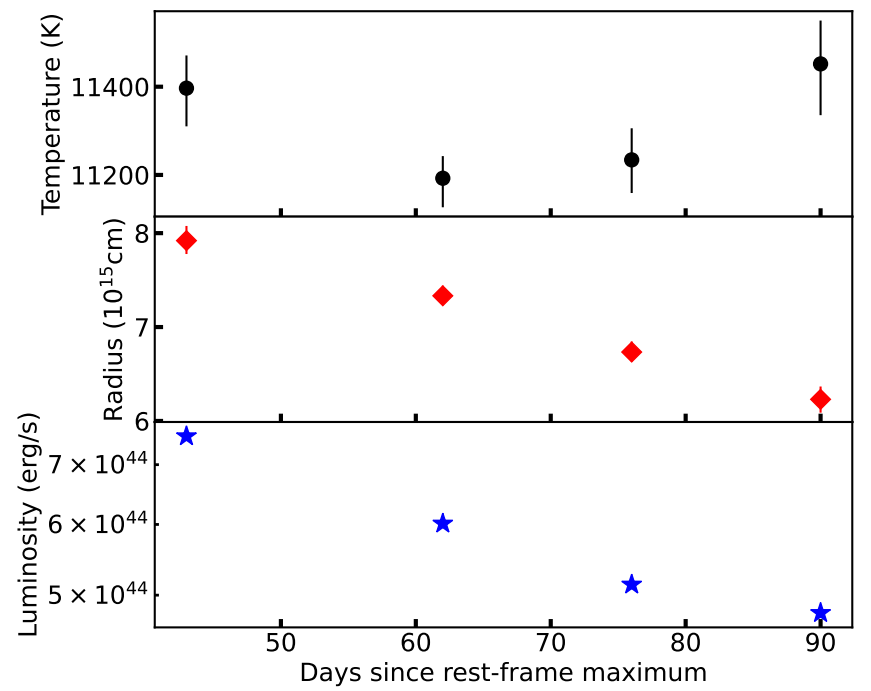

Fig. B.2. The evolution of UV/optical emission of CSS1029 at different epochs. From top to bottom panel, we show the evolution of blackbody luminosity, temperature and radius, respectively, which are derived from blackbody fittings to the Swift UVOT data. 